\title{
Notch-effector CSL promotes squamous cell carcinoma by repressing histone demethylase KDM6B
}

\author{
Dania Al Labban, ${ }^{1}$ Seung-Hee Jo, ${ }^{2}$ Paola Ostano, ${ }^{3}$ Chiara Saglietti, ${ }^{4}$ Massimo Bongiovanni, ${ }^{4}$ \\ Renato Panizzon, ${ }^{5}$ and G. Paolo Dotto ${ }^{1,2}$ \\ 'Department of Biochemistry, University of Lausanne, Epalinges, Switzerland. 'Cutaneous Biology Research Center, Massachusetts Ceneral Hospital, Charlestown, Massachusetts, USA. ${ }^{3}$ Cancer Cenomics \\ Laboratory, Edo and Elvo Tempia Valenta Foundation, Biella, Italy. ${ }^{4}$ Department of Pathology, and ${ }^{5}$ Department of Dermatology, Lausanne University Hospital (CHUV), Lausanne, Switzerland.
}

Notch 1/2 genes play tumor-suppressing functions in squamous cell carcinoma (SCC), a very common malignancy in skin and internal organs. In contrast with Notch, we show that the transcription factor CSL (also known as RBP-J $\kappa$ ), a key effector of canonical Notch signaling endowed with intrinsic transcription-repressive functions, plays a tumor-promoting function in SCC development. Expression of this gene decreased in upper epidermal layers and human keratinocytes (HKCs) undergoing differentiation, while it increased in premalignant and malignant SCC lesions from skin, head/neck, and lung. Increased CSL levels enhanced the proliferative potential of HKCs and SCC cells, while silencing of CSL induced growth arrest and apoptosis. In vivo, SCC cells with increased CSL levels gave rise to rapidly expanding tumors, while cells with silenced CSL formed smaller and more differentiated tumors with enhanced inflammatory infiltrate. Global transcriptomic analysis of HKCs and SCC cells with silenced CSL revealed major modulation of apoptotic, cell-cycle, and proinflammatory genes. We also show that the histone demethylase KDM6B is a direct CSL-negative target, with inverse roles of CSL in HKC and SCC proliferative capacity, tumorigenesis, and tumor-associated inflammatory reaction. CSL/KDM6B protein expression could be used as a biomarker of SCC development and indicator of cancer treatment.

\section{Introduction}

Squamous cell carcinomas (SCCs) are the most common forms of human cancer (1) and are a major cause of death. These heterogeneous tumors originate from surface epithelia, such as skin and aerodigestive and urogenital tracts, in response to a variety of noxious conditions (1). Common gene alterations are found in these tumors, distinguishable in 2 main categories: mutations with driver function in many cancer types and more selective mutations affecting squamous cell-fate commitment and/or ensuing terminal differentiation (1).

Notch signaling is an important form of direct cell-cell communication with an essential role in the switch between proliferation and differentiation of keratinocytes (2). It impinges on direct regulators of proliferative potential, such as p21WAF1/Cip1 (3) and p63 (4), as well as more indirect paracrine mechanisms involved in normal skin homeostasis and barrier function (5-7). This pathway plays a similarly important role in oral (8), esophageal (9), bronchial (10), and cervical epithelia (11).

Inactivating mutations of NOTCH1, NOTCH2, and NOTCH3 receptor genes are frequently found in SCCs from various body sites (1). Of these, NOTCH1 plays a prominent role in SCC suppression. In addition to being frequently mutated, this gene is a direct positive target of p53 in keratinocytes and is commonly downmodulated as a consequence of compromised p53 function in SCC cells $(12,13)$.

Conflict of interest: The authors have declared no conflict of interest exists.

Submitted: August 16, 2017; Accepted: March 20, 2018.

Reference information: J Clin Invest. 2018;128(6):2581-2599.

https://doi.org/10.1172/JCI96915.
In spite of its highly context-dependent functions, "canonical" Notch signaling has been highly conserved throughout evolution, with translocation of activated Notch intracellular domain into the nucleus, converting the DNA-binding protein CSL (RBP-Jк) from a repressor into an activator of transcription (14). While functioning as an essential mediator of Notch activation, CSL is endowed with intrinsic transcription-repressive function and can be converted by other cofactors, besides $N O T C H$, into an activator $(15,16)$. Evidence from various genetic models indicates that abrogation of CSL function can have consequences beyond suppression of Notch signaling, while conversely, NOTCH activation may involve other mediators besides CSL $(15,16)$. In skin, the more pronounced phenotype of mice with keratinocyte-specific deletion of NOTCH1 and NOTCH2 genes versus CSL suggests that NOTCH has a broader function than CSL (17). However, the converse possibility that CSL also plays a role distinct from that of NOTCH in keratinocytes and SCC development has not yet been addressed.

Epigenetic regulators impinge on the balance between cancer cell renewal and commitment to differentiation (18). The cumulative mutation rate of this family of genes in SCCs is more than $50 \%$, with a number of them involved in a squamous differentiation program (1). Trimethylation of histone $\mathrm{H} 3$ at lysine 27 (H3K27me3) is a key histone modification, with transcription-repressive function that is deregulated in a variety of cancers (19). Removal of H3K27me2 and H3K27me3 marks by the KDM6B (JMJD3) demethylase can operate in concert with NOTCH activation in cancer development, as indicated by the shared prooncogenic role of the 2 genes in T cell acute lymphoblastic leukemia (T-ALL) cells (20). Like NOTCH, KDM6B is involved in key cellular processes, such as 
cell-cycle control, senescence, and differentiation, and may play a role in cancer development in a context-dependent manner (2123). In fact, $K D M 6 B$ expression is upregulated in several malignancies, such as Hodgkin's lymphoma (24), breast cancer (25), gliomas (26), melanoma (27), and renal cell carcinoma (28), while it is suppressed in others, including lung adenocarcinoma and squamous cell carcinoma (29), colon cancer $(30,31)$, and liver and pancreatic cancers (32). While inactivating KDM6B gene mutations are only found in 1\%-3\% of SCCs (cBioPortal for Cancer Genomics; http:// www.cbioportal.org), the gene, like NOTCH1, has been implicated as a positive determinant of squamous cell differentiation (33).

Increased immune cell infiltration into tumors is a positive prognostic sign for elimination of cancer cells and favorable response to cancer immunotherapy (34). In cells of the immune/ inflammatory systems, a crosstalk has been uncovered between KDM6B and NF-KB and STAT family members through a variety of mechanisms, including physical association (35-38). In contrast, mechanisms involved in the control of $K D M 6 B$ in keratinocytes and SCC and possible interconnection with NOTCH/CSL signaling have not been investigated.

We report here that, in contrast with NOTCH activation, CSL has an intrinsic tumor-promoting function in SCC development that is mediated, in part, by suppression of $K D M 6 B$ expression, a direct $C S L$ target. Compared with $C S L, K D M 6 B$ has an inverse effect on HKC and SCC proliferative capacity, tumorigenesis, and tumor-associated inflammatory reaction, and the 2 proteins could be used as prognostic markers of SCC development.

\section{Results}

CSL gene expression is reduced in differentiating keratinocytes and induced in premalignant and malignant cancer cells. CSL can play biologically significant roles as a repressor of transcription independently of NOTCH activation (15). Control of CSL function in this context can occur through modulation of its expression (39, 40). In human skin, immunofluorescence analysis showed that CSL is highly expressed in keratinocytes of lower epidermal layers, while it is substantially downmodulated in upper layers (Figure 1A and Supplemental Figure 1A; supplemental material available online with this article; https://doi.org/10.1172/JCI96915DS1), in contrast with the opposite pattern of NOTCH $1 / 2$ expression that we previously reported (41). Similarly elevated CSL expression was also found in lower layers of oral epithelium, with pronounced downmodulation in upper layers (Figure 1B).

Actinic keratoses (AK) are premalignant SCC precursor lesions associated with an altered squamous cell differentiation program and expansion of cells of the proliferative compartment. Immunohistochemical analysis of these lesions showed pronounced upregulation of CSL expression relative to the surrounding normal epidermis (Figure 1C and Supplemental Figure 1B). Upregulated CSL expression was also found in dysplastic versus normal epithelium of the oral cavity, with a further increase in adjacent SCCs (Figure 1D and Supplemental Figure 1C). In parallel, immunohistochemical analysis of tissue arrays of skin and oral SCCs showed significantly greater CSL signal intensity in high- versus low-grade tumors (Figure 1E).

These findings were mirrored by a pattern of CSL expression in cultured cells. In 3 independent strains of primary human kera- tinocytes (HKCs), expression of the CSL protein was substantially reduced with induction of differentiation by high-confluency conditions or suspension culture at times when a terminal differentiation marker such as involucrin was induced (Figure 2A and Supplemental Figure 1D). Downmodulation of CSL protein levels upon differentiation was likely due to a posttranscriptional mechanism, as CSL mRNA levels were increased rather than decreased (Figure 2B and Supplemental Figure 1E).

Relative to HKCs, CSL protein levels were increased in a panel of skin and oral SCC cell lines, independently of p53 status (The TP53 Web Site, http://p53.free.fr) (42) (Figure 2C). mRNA levels were also higher in most of the tested cancer cell lines, indicating that CSL expression can be deregulated at both transcriptional and posttranscriptional levels (Figure 2D). A similar increase in CSL protein levels, with less consistent upregulation of mRNA levels, was also found in a panel of lung SCC cells relative to primary human bronchial epithelial cells (HBECs) (Figure 2, E and F). Upregulation of CSL expression in SCC13 cells versus HKCs was also detected by immunohistochemical staining of these cells brought into suspension, using the same fixation and staining procedures utilized in the clinic for analysis of cytological specimens (Supplemental Figure 1F).

CSL is a positive regulator of keratinocyte and SCC proliferative potential. To assess biological significance of the above findings, we evaluated consequences of modulated CSL expression in HKCs. Three independent strains were stably infected with a retrovirus expressing myc-tagged CSL, which in several previous papers was shown to be functionally equivalent to untagged CSL $(43,44)$. A several-fold increase of CSL levels (Figure 3A), approximating that in SCC cells, resulted in significantly enhanced proliferative activity, as assessed by an assay for metabolically active cells (CellTiterGlo) (Figure 3B). Clonogenicity assays provide a well-established method to assess the fraction of keratinocytes with elevated proliferative potential (putative stem cells) (45). Paralleling previous results, increased CSL expression significantly enhanced the fraction of HKCs with clonogenic capacities (Figure 3C).

Conversely, overall proliferation of HKCs was strongly suppressed by shRNA-mediated CSL gene silencing (Figure 3, D and E), associated with reduction of their clonogenic potential (Figure $3 \mathrm{~F}$ ). The sphere-forming capability of cells embedded in Matrigel was used as an additional measure of the proliferative potential of cells (46). Strong inhibitory effects were also observed with this assay upon CSL silencing in all tested HKC strains (Figure 3G). Decreased proliferative potential of HKCs was paralleled by decreased DNA synthesis, as assessed by EdU labeling of cells (Figure 3H).

Like HKCs, SCC cell lines contain subpopulations with elevated proliferative potential (putative cancer stem cells), which can be assessed by clonogenicity assays (47). Lentivirus-mediated increase of CSL expression significantly enhanced colony- and sphere-forming capability of a panel of SCC cell lines (Figure 4, A-C), while conversely, CSL gene silencing inhibited clonogenic potential and reduced overall proliferation (Figure 4, D-F). Growth inhibitory effects of CSL gene silencing were not due to specific toxic mechanisms, as they were not observed with SCCO 28 cells (Figure 4, D-F), an oral SCC-derived cell line with advanced features of epithelial-to-mesenchymal transformation (48). The sphere-forming capability of various SCC cell lines in 
A

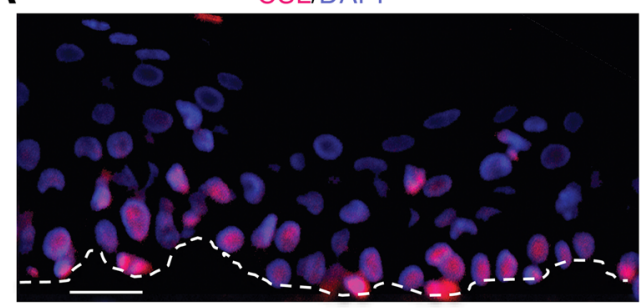

B

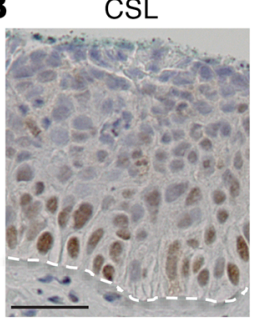

C

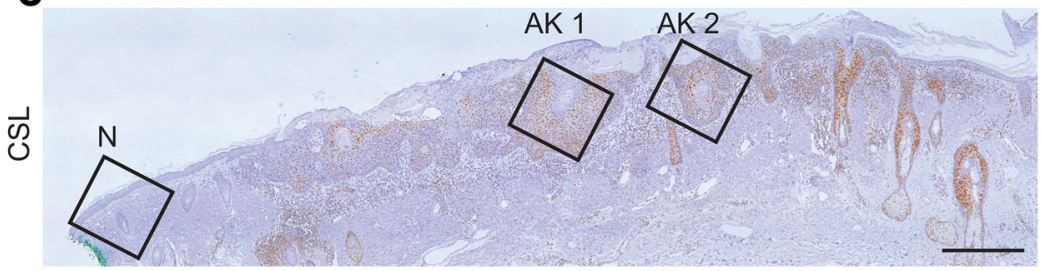

$\mathrm{N}$

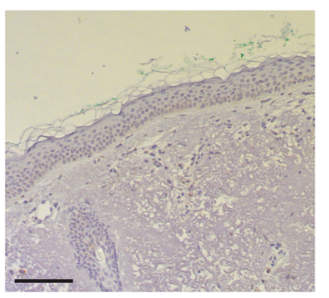

AK 1
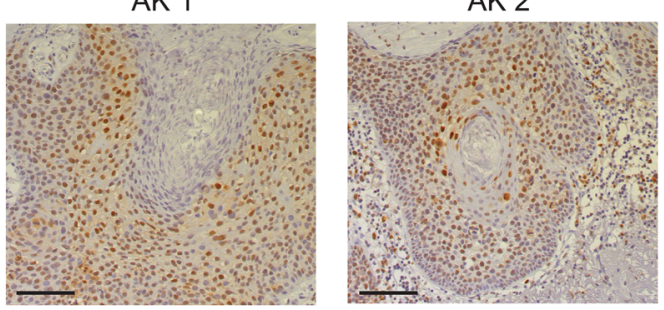

D

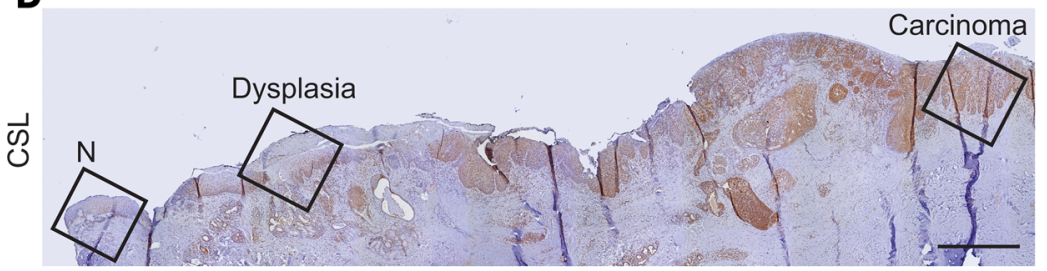

$\mathrm{N}$

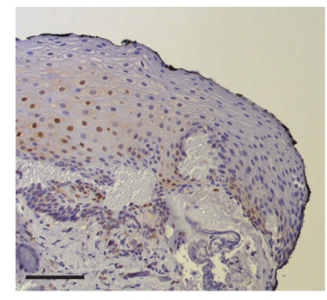

Dysplasia
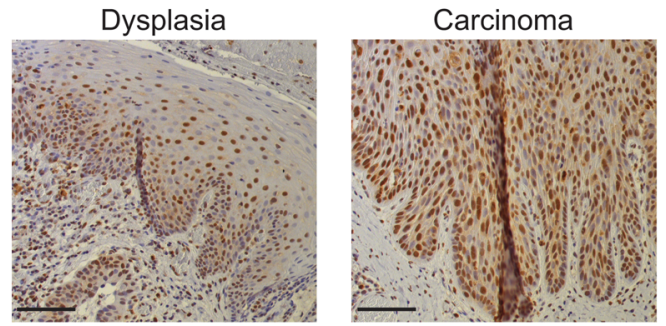

Figure 1. Higher CSL expression in premalignant and malignant squamous cancer lesions. (A) Immunofluorescence analysis of CSL expression in epidermis of normal skin, with DAPI staining for cell identification. Similar analysis of normal skin samples from 2 more individuals is shown in Supplemental Figure 1A. $n=3$ individuals. Scale bar: 25 $\mu \mathrm{m}$. (B) Immunohistochemical analysis of CSL expression in normal oral epithelium. $n=1$ individual. Scale bar: 125 $\mu \mathrm{m}$. Dotted lines mark the border of the epidermal compartment relative to the underlying mesenchyme. (C) Immunohistochemical analysis of CSL expression in an AK (54) lesion and flanking normal (N) skin. Similar analysis of additional AK lesions and flanking skin is shown in Supplemental Figure 1B. $n=5 \mathrm{AK}$ regions; $n=5 \mathrm{~N}$ regions. Right panel: CSL signal quantification of all AK patients. Data are shown as mean \pm SEM, 2-tailed paired $t$ test. Scale bars: $1000 \mu \mathrm{m}$ (upper panels); $100 \mu \mathrm{m}$ (lower panels). (D) Immunohistochemical analysis of CSL expression in oral SCC lesions and flanking dysplastic and normal tissue. For additional lesions, see Supplemental Figure $1 \mathrm{C} . n=7$ carcinoma; $n=4$ dysplastic; $n=7$ normal regions. Right panel: CSL signal quantification of all SCC patients. Data are shown as mean \pm SEM, 1-way ANOVA with Dunnett's test. Scale bars: $1000 \mu \mathrm{m}$ (upper panels); $100 \mu \mathrm{m}$ (lower panels). (E) Immunohistochemical analysis of CSL expression in tissue arrays of skin and oral SCC lesions in low- versus high-grade tumors (defined by invasion beyond submucosal tissue). Representative images are shown along with CSL signal quantification. Data are shown as mean \pm SEM, 2-tailed unpaired $t$ test. $n=8$ low-grade skin lesions; $n=12$ high-grade skin lesions; $n=18$ low-grade oral lesions; $n=8$ high-grade oral lesions. Scale bars: $200 \mu \mathrm{m}$. (C-E) ${ }^{* *} P<0.005$; ${ }^{* * *} P<0.0005$.
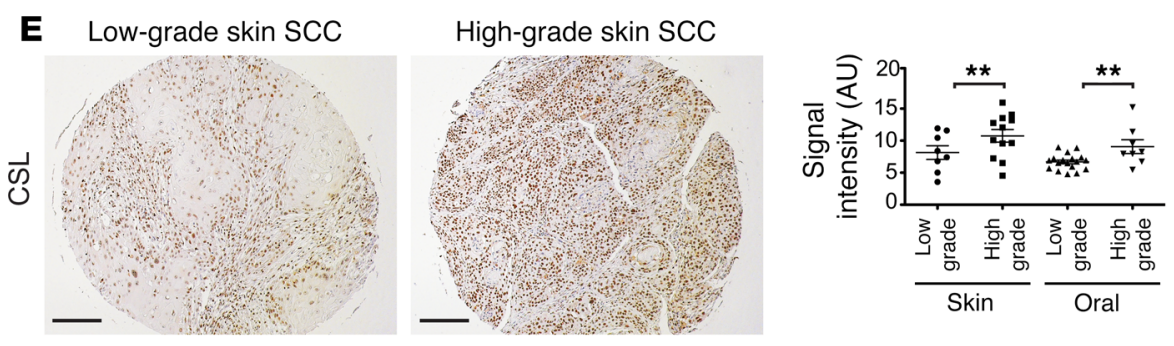

Matrigel suspension was also suppressed by CSL silencing (Figure $4 \mathrm{G}$ ), and decreased proliferation of SCC cells could be explained by decreased DNA synthesis as well as enhanced apoptosis (Supplemental Figure 2, A and B).

CSL promotes SCC tumor formation in a xenograft model. We next assessed the effects of CSL modulation on the tumorigenic potential of SCC cells utilizing an orthotopic ear-injection assay in immune-compromised mice (40). CSL-overexpressing SCC cells formed rapidly expanding tumors, and mice were sacrificed at early times, in most cases prior to tumor formation by control cells injected in parallel (Figure 5A). Conversely, tumor formation by SCC cells was substantially delayed by CSL silencing and even in some cases totally suppressed (Figure 5B). Decreased tumor formation was mirrored by decreased cellular proliferation, as 
A

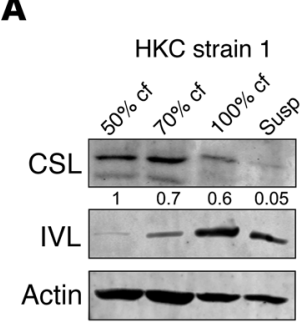

B

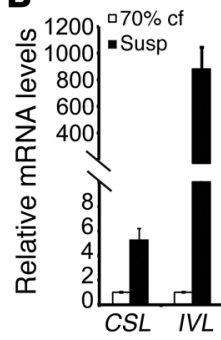

C

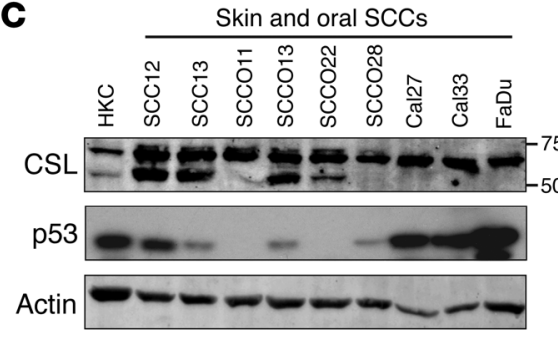

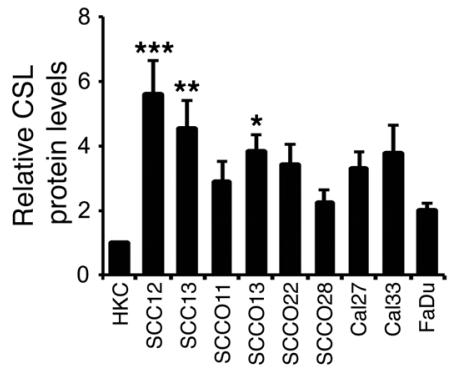

$\mathbf{F}$
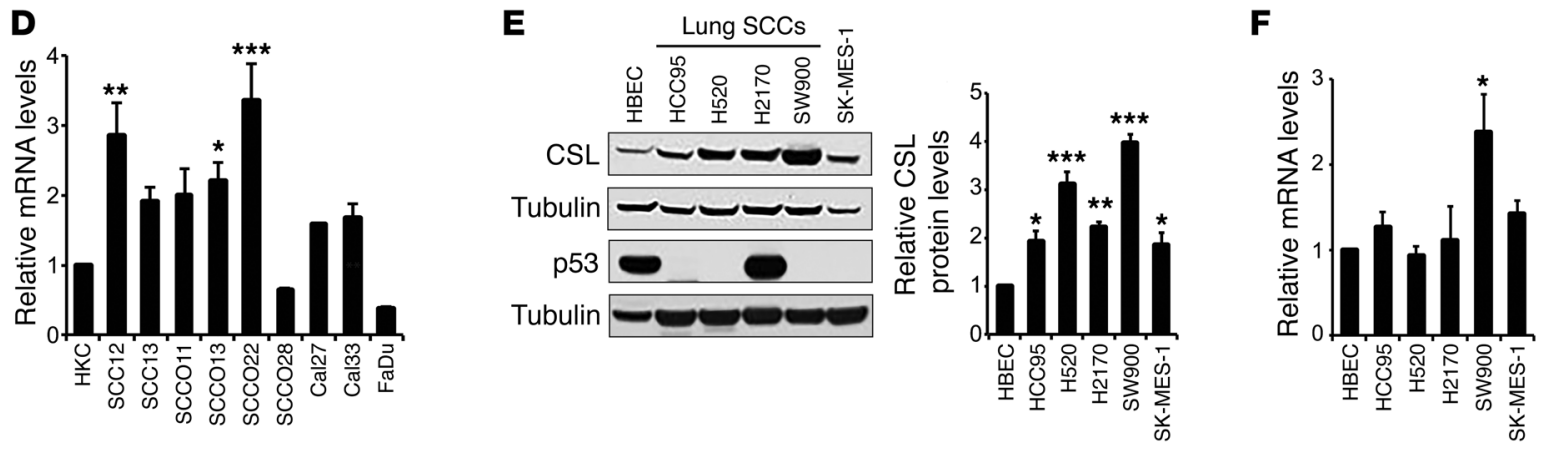

Figure 2. Higher CSL expression in proliferating vs. differentiating keratinocytes and squamous cancer cells. (A) Immunoblot analysis of CSL expression in HKCs under proliferating (50\% and 70\% confluence [cf]) versus differentiating culture conditions ( 7 days at $100 \%$ cf; 48 hours in suspension culture [susp]). Immunoblot was sequentially probed with antibodies against CSL, involucrin (IVL) (80), and actin. Numbers refer to relative folds of CSL expression using actin for normalization. Results of similar experiment with 2 other HKC strains are shown in Supplemental Figure 1D. (B) HKCs as in $\mathbf{A}$ were analyzed by RT-qPCR for CSL and involucrin mRNA expression with 36B4 for normalization. Similar analysis of 2 additional HKC strains is shown in Supplemental Figure 1E. (C) Immunoblot analysis of CSL expression in HKCs under proliferating conditions versus panel of skin and oral SCC cell lines. CSL protein on immunoblot can be variously detected as a single or dual band, probably reflecting different phosphorylation states, with no correlation to p53 status of cells. Immunoblot was sequentially probed with antibodies against CSL, actin, and p53. Right panel: quantification of relative levels of CSL expression of this and 2 other immunoblots, using actin for normalization. (D) Cells as in C were analyzed by RT-qPCR for CSL mRNA expression with 36B4 for normalization. (E) Lung SCC cell lines were analyzed in parallel with HBECs for levels of CSL or p53 expression utilizing tubulin as equal loading control. Right panel: quantification of relative levels of CSL expression of this and 2 other immunoblots. (F) Cells as in E were analyzed by RT-qPCR for CSL mRNA expression with 36B4 for normalization. (C-F) Data are shown as mean $\pm \mathrm{SEM} .{ }^{*} P<0.05 ;{ }^{* *} P<0.005 ;{ }^{* *} P<0.0005,1$-way ANOVA with Dunnett's test. $n=3$ independent experiments.

assessed by Ki67 positivity (Figure 6A), and enhanced differentiation, as assessed by keratin 1 (K1) and K10 expression (Figure 6, B and C). Importantly, despite the lack of intrinsic effects on the proliferation of SCCO28 cells, CSL silencing also suppressed tumorigenicity of these cells, with decreased Ki67 positivity (Figure 5B and Figure 6A). The decreased tumorigenicity was accompanied in all cases by enhanced infiltration of immune cells together with a marked increase in expression of the proinflammatory cytokine IL6 (Supplemental Figure 3, A and B).

CSL acts as a shared determinant of gene expression in HKCs and SCC cells. Independently of NOTCH activation, CSL plays a constitutive repressive function in gene transcription. By RNAsequencing (RNA-seq) analysis, more than 1,000 genes were consistently up- or downregulated in HKCs by CSL gene silencing (NCBI's Gene Expression Omnibus database; GEO GSE102762). Gene ontology (GO) analysis showed that gene families most significantly upregulated by CSL silencing are related to apoptosis and regulation of programmed cell death, while those most significantly downregulated are related to mitosis and DNA replication and repair (Tables 1 and 2 and Supplemental Table 1). By gene set enrichment analysis (GSEA) (49), we found no significant correlation between CSL-modulated genes and previously established
NOTCH1 and differentiation signature genes in HKCs (4), further consistent with a NOTCH separate function of CSL in these cells (Table 3 and Supplemental Table 2). In contrast, there was significant correlation between CSL-modulated genes and gene signatures of pathways implicated in inhibition of keratinocyte proliferation (specifically NF-KB and TGF-B) and inflammation (Figure 7A and Table 3).

Similar RNA-seq analysis was carried out in SCC13 cells with CSL gene silencing (GEO GSE102762). As with HKCs, many genes were deregulated upon CSL silencing in SCC13 cells, with a few hundred that were similarly modulated in both (Figure 7B). There was also high concordance in GO analysis, which showed that mitosis and DNA replication genes were among those downregulated by CSL silencing in SCC13 cells and that apoptosis genes were among those upregulated in response to CSL silencing (Tables 1 and 2, and Supplemental Table 1). GSEA also showed a similar positive correlation between the expression profiles of SCC13 cells with CSL gene silencing and gene signatures related to TGF-B, NF- $\mathrm{kB}$, TNF-A, INF, and inflammatory responses, while there was again no significant association with $\mathrm{NOTCH}$ or keratinocyte differentiation gene signatures (Table 3 and Supplemental Table 2). Quantitative reverse-transcriptase PCR (RT-qPCR) 
A

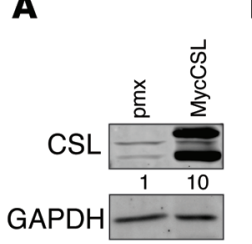

B

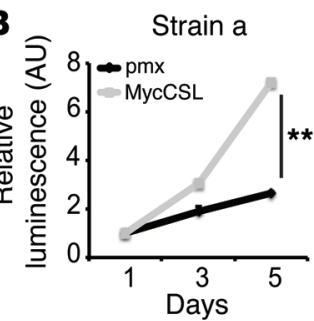

Strain b

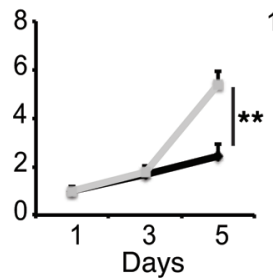

Strain c
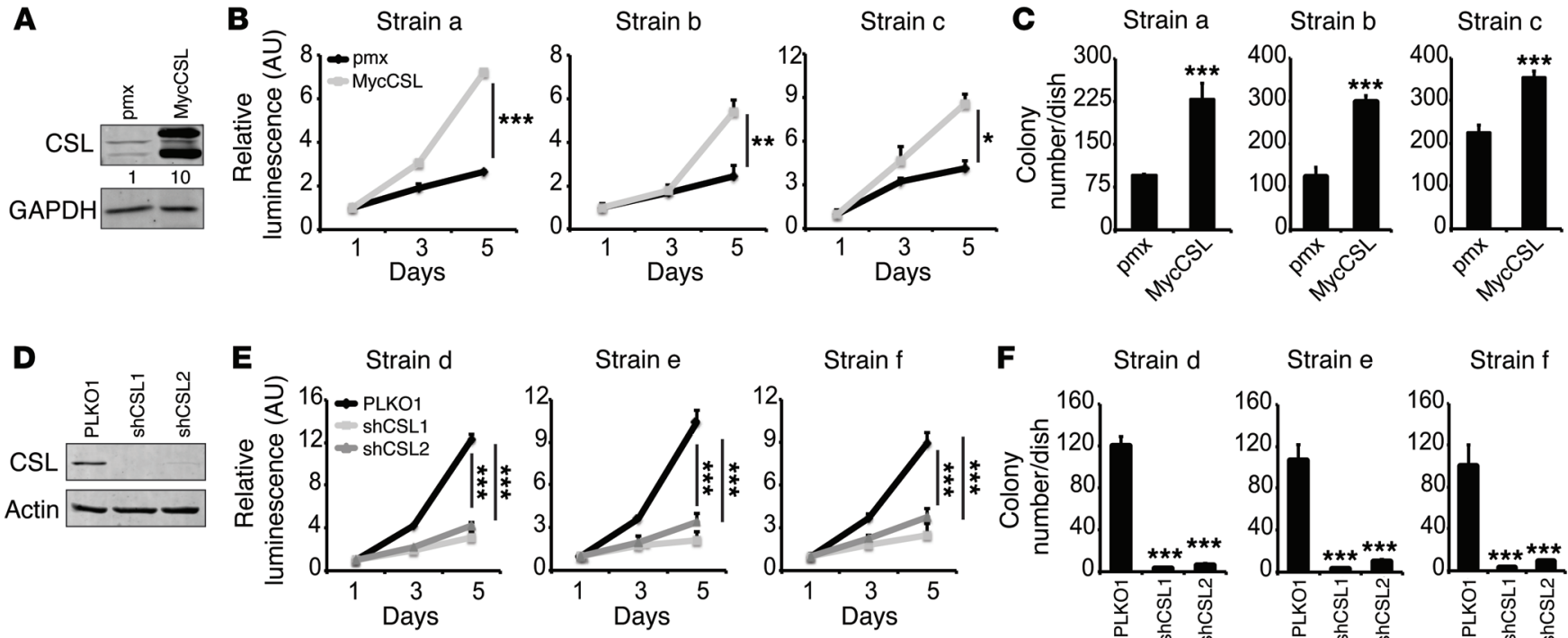

$\mathbf{F}$

Strain d

Strain e

Strain $\mathrm{f}$
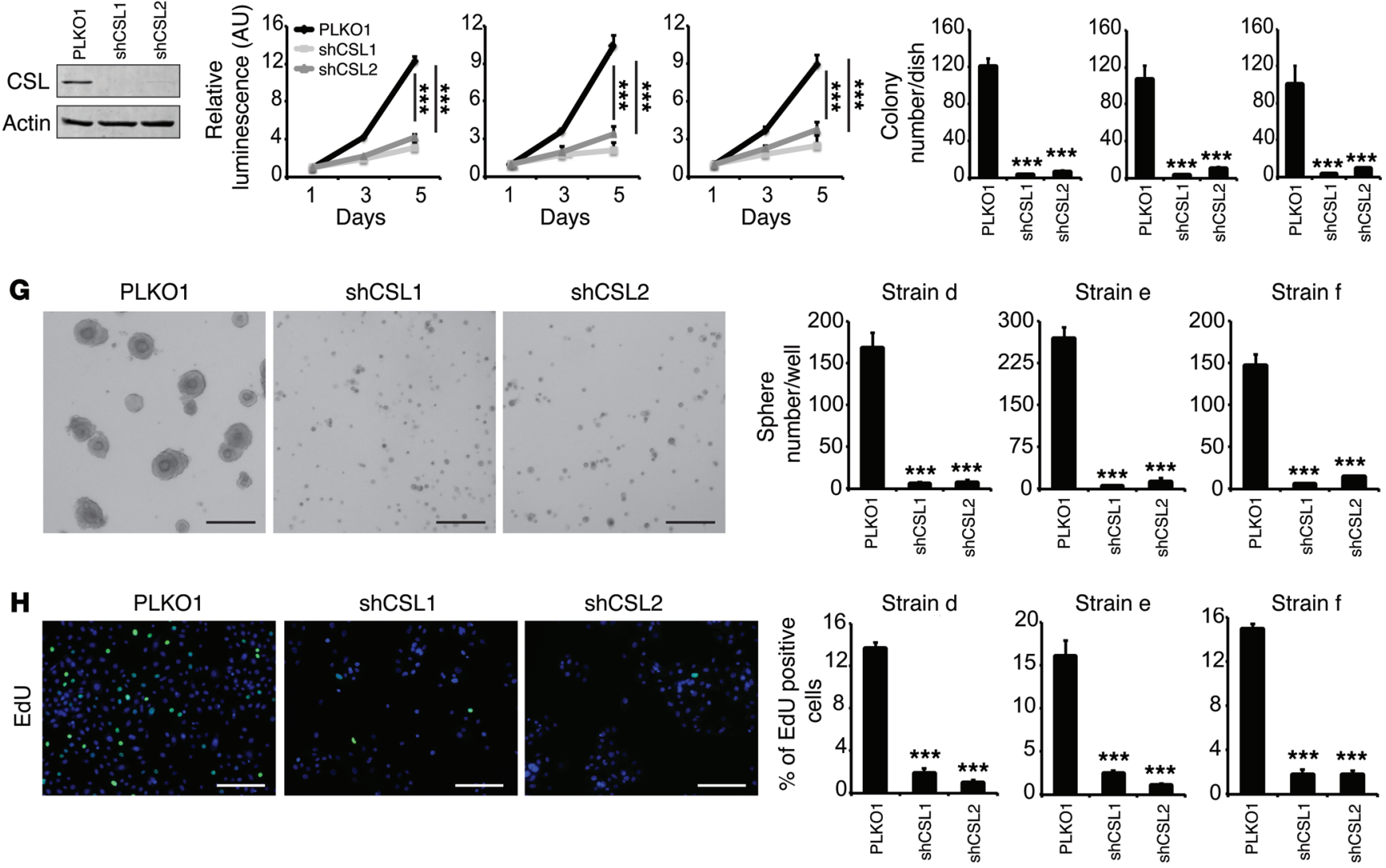

Figure 3. Positive role of CSL in promoting keratinocyte proliferative potential and cell survival. (A) HKCs infected with retroviral vector for constitutive Myc-tagged CSL expression (mycCSL) in parallel with vector control $(\mathrm{pmx})$, were analyzed for CSL expression by immunoblotting. Numbers refer to relative folds of CSL expression using GAPDH for normalization. (B) Three independent HKC strains, infected as in A, were tested for cell metabolic activity assays. Results are presented as luminescence intensity values relative to day 1. (C) The same HKC strains as in B were plated at limited density, and colony formation was measured. (D) HKCs infected with 2 shRNA lentiviruses against CSL (shCSL1 and shCSL2) versus empty control (PLKO1) were analyzed for CSL expression by immunoblotting. (E) Three HKC strains infected as in D were tested for cell metabolic assays. Lower luminescence/metabolic activity rates of control cells in panel $\mathbf{B}$ versus $\mathbf{E}$ are likely due to the use of cells with higher passage number in $\mathbf{B}$, which was necessary because of lower efficiency of infection with PMX vectors used for those experiments. (F) The same HKC strains as in $\mathbf{E}$ assessed for colony formation. (C) The same HKC strains as in $\mathbf{E}$ were plated in Matrigel suspension cultures. The number of spheroids was counted using Imagel software (NIH). Shown are representative images of spheroids formed by 1 HKC strain together with quantification of 3 strains. Scale bars: $250 \mu \mathrm{m}$. (H) The same HKC strains as in E were labeled with EdU for 6 hours. EdU-positive cells were counted using Imagel software. Shown are representative images of cells of $1 \mathrm{HKC}$ strain together with quantification of 3 strains. Scale bars: $100 \mu \mathrm{m}$. (B and C) Data are shown as mean \pm SD, 2-tailed unpaired $t$ test. $n=3$ biological replicates/strain. (E-H) Data are shown as mean \pm SD. ${ }^{*} P<0.05$; ${ }^{* *} P<0.005 ;{ }^{* *} P<0.0005$, 1-way ANOVA with Dunnett's test. $n=3$ biological replicates/strain.

analysis confirmed downregulation of the panel of cell-cycle regulatory genes and upregulation of apoptotic and proinflammatory genes in HKCs and SCC13 cells as well as other SCC cells upon CSL silencing (Figure 7, C-E).

To probe into the consequences of CSL gene silencing in cancer cells that are resistant to its growth-inhibitory effects, similar RNA-seq analysis was carried out with SCCO28 cells (GEO
GSE102762). In striking contrast to HKCs and SCC13 cells, only 36 genes were upregulated in SCCO28 cells upon CSL silencing and 4 were downmodulated. These genes belonged to families related to membrane/signaling, enzymes, extracellular matrix, and inflammatory/immunomodulatory cytokines, but none were directly involved in cell division control or apoptosis (Figure 7F and Supplemental Table 1). 
A

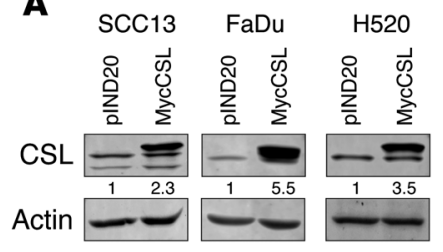

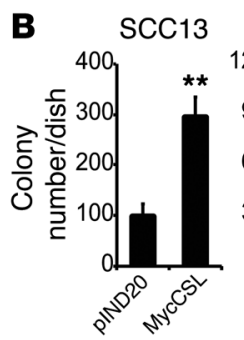
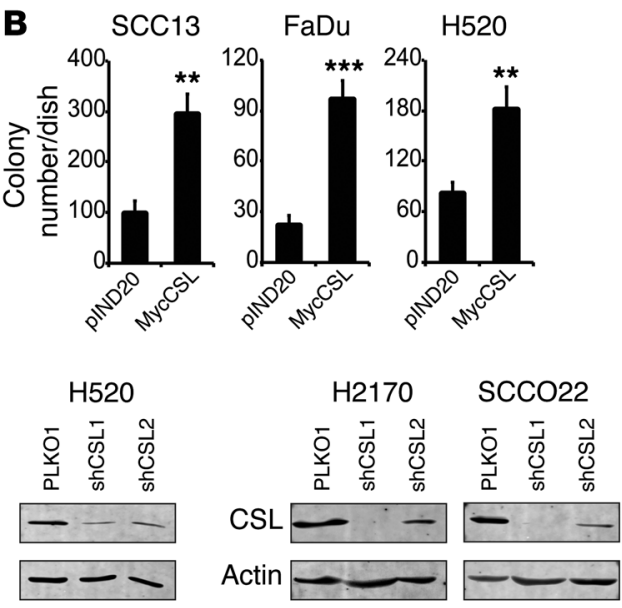
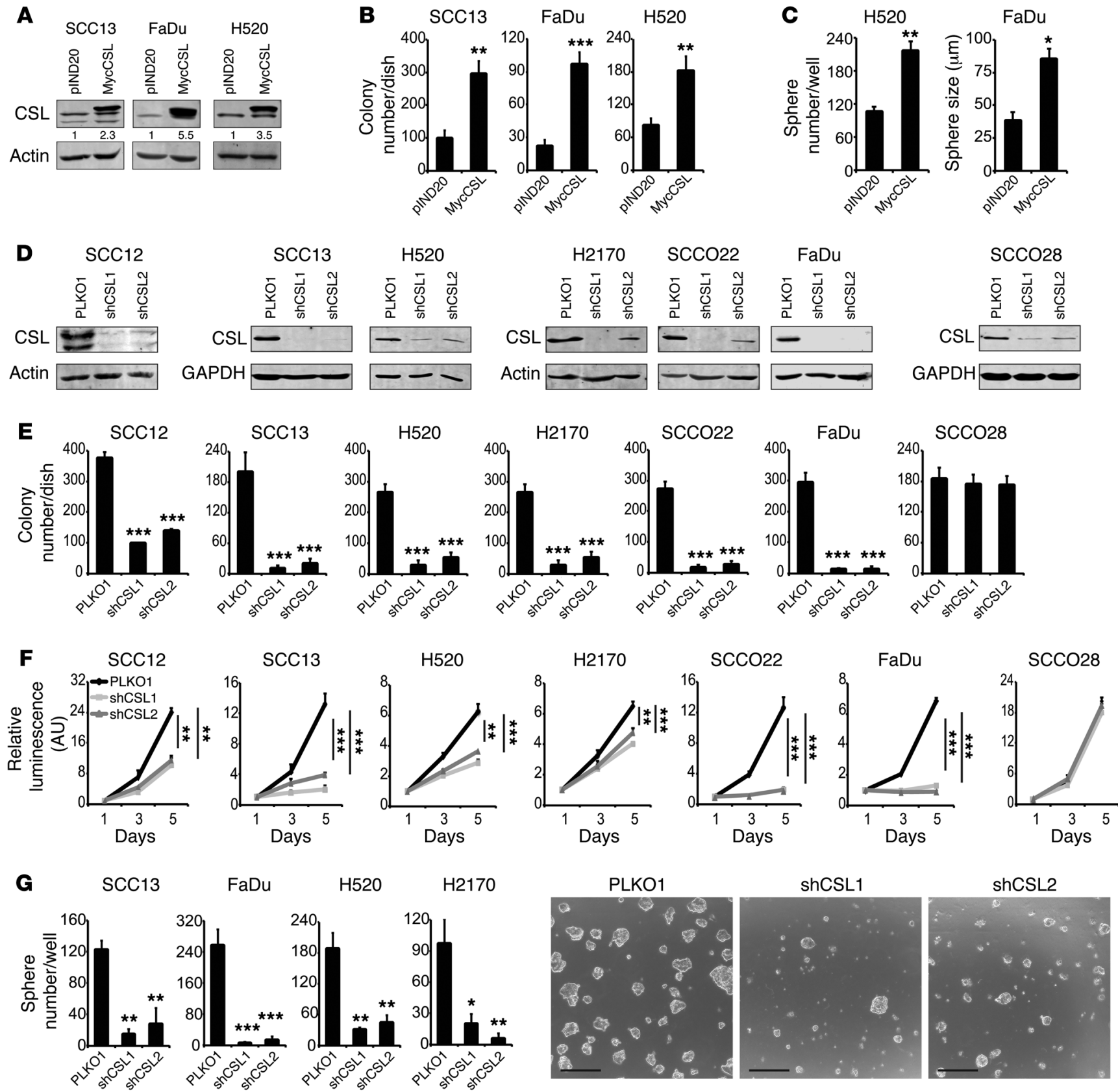

H520

$\mathrm{H} 2170$
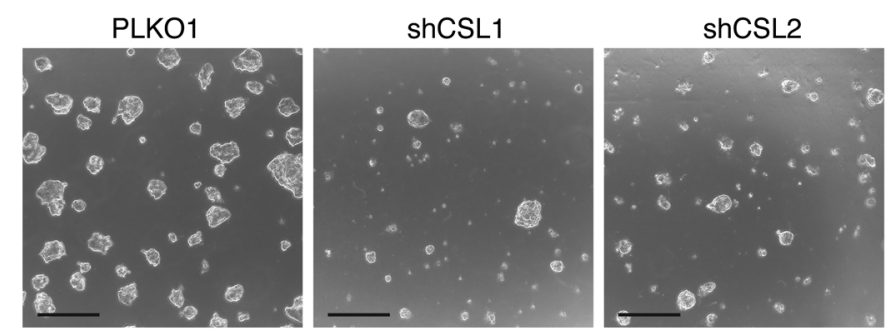

Figure 4. Positive role of CSL in promoting proliferative potential of skin, oral, and lung SCC cells. (A) Cell lines derived from skin (SCC13), oral (FaDu), and lung (H520) SCCs stably infected with lentiviral vector for inducible Myc-tagged CSL versus empty vector control (pIND20) were analyzed 2 days after doxycycline treatment for CSL expression by immunoblotting. Numbers refer to relative folds of CSL expression using actin for normalization. (B) The same SCC cells as in A were plated at a limited density, and colony formation was measured. (C) The same SCC cells as in B were plated in Matrigel suspension. Number and size of spheroids were assessed using Image) software. (D) Cell lines derived from skin (SCC12, SCC13), oral (SCCO22, SCCO28, and FaDu), and lung (H520 and H2170) SCCs infected with 2 shRNA lentiviruses against CSL were analyzed for CSL expression by immunoblotting. (E) The same SCC cells as in D were plated at a limited density, and colony formation was measured. (F) The same SCC cells as in E were tested for cell metabolic activity assays. (C) The same SCC cells as in $\mathbf{E}$ were plated in Matrigel suspension. Shown are representative images of spheroids formed by FaDu cells. Scale bars: $250 \mu \mathrm{m}$. (B and C) Data are shown as mean \pm SEM, 2-tailed unpaired $t$ test. $n=3$ independent experiments. (E-G) Data are shown as mean \pm SEM. ${ }^{*} P<0.05 ;{ }^{* *} P<0.005 ;{ }^{* *} P<0.0005,1$-way ANOVA with Dunnett's test. $n=3$ independent experiments.

IL6 was among the proinflammatory cytokines upregulated in SCC13, SCCO22, and SCCO28 cells with silenced CSL, consistent with increased IL6 expression found in small tumors formed by these cells by ear injection (Supplemental Figure 3B). However, this could be a secondary event, as tumor formation by these assays took a relatively long time and, in a number of cases, cells with silenced CSL yielded no detectable tumors. An important question was therefore whether increased expression of IL6 and other coregulated cytokines occurs in SCC cells with silenced CSL even in vivo at early times preceding drastic differences in tumor formation. To 
A
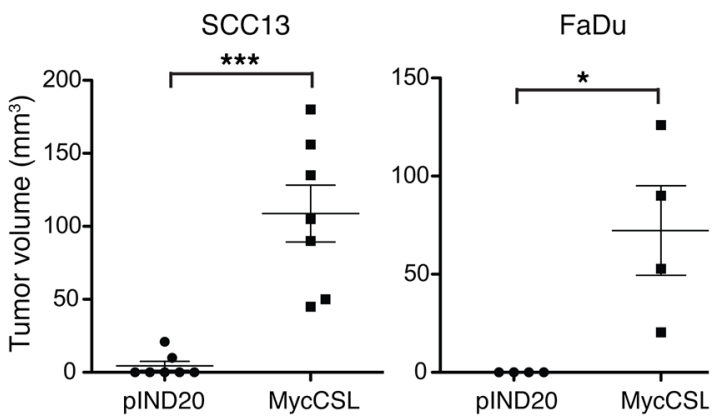
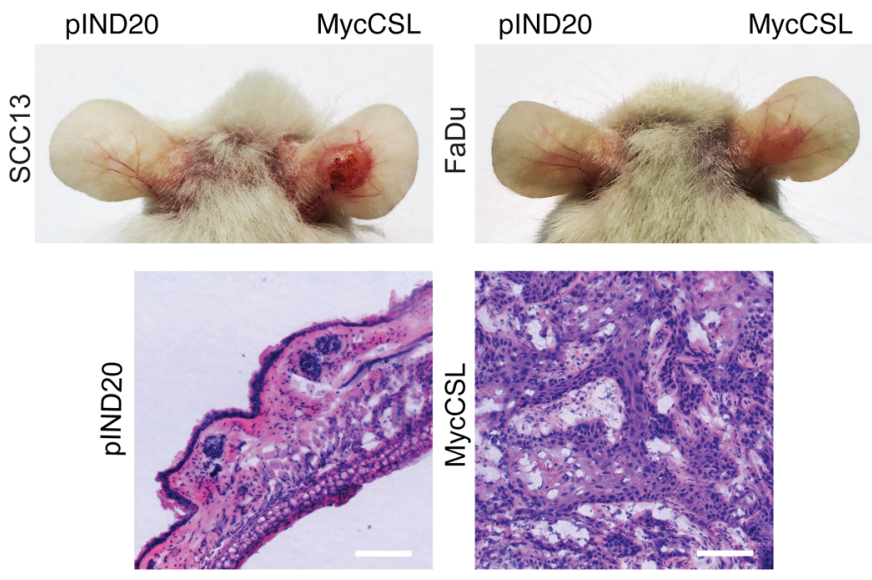

B

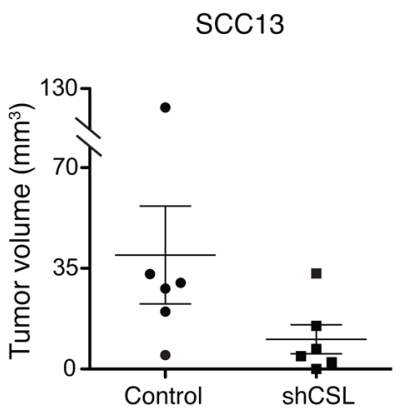

PLKO1

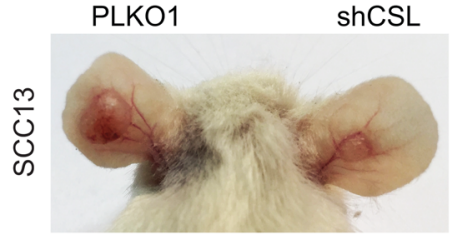

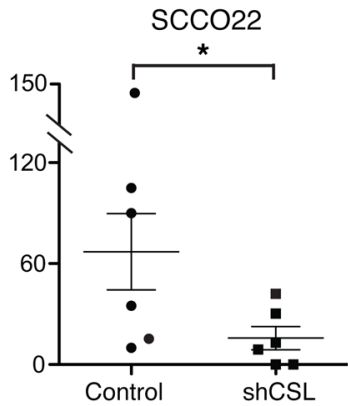

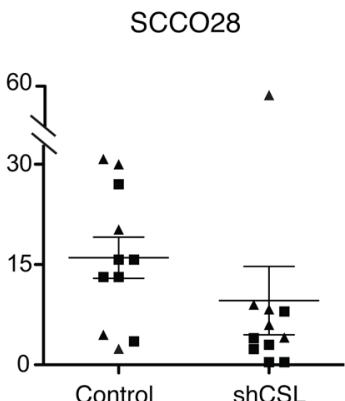

- Exp. 1

- Exp. 2

PLKO1
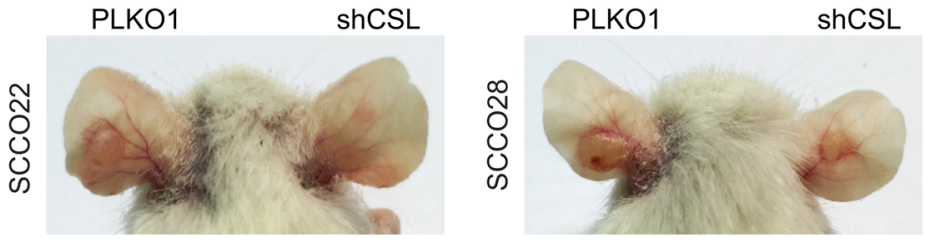

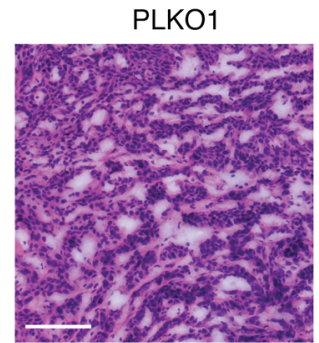

shCSL

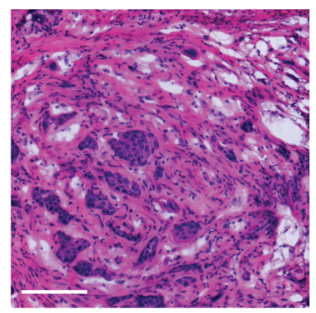

Figure 5. Tumor modulatory effects of increased versus suppressed CSL expression on SCC cells. (A) SCC13 and FaDu cells stably infected with lentiviral vector for doxycycline-inducible Myc-tagged CSL or empty vector control (pIND20) were injected in parallel into ears of NOD/SCID mice. Shown are tumor volumes at day of sacrifice (left panel), with representative images for 1 pair of mouse ears (right panel) and corresponding H\&E staining of SCC13 lesions. $n=7$ mice for SCC13 lesions; $n=4$ mice for FaDu lesions. (B) SCC13, SCCO22, and SCCO28 cells infected with CSL-silencing lentivirus versus empty vector control were injected into ears of NOD/SCID mice. Shown are tumor volumes (upper panels) with representative images of 1 pair of mouse ears (lower panels), and corresponding H\&E staining of SCCO22 lesions (right panels). Scale bars: $250 \mu \mathrm{m}$. $n=6$ mice for SCC13 lesions; $n=6$ mice for SCCO22 lesions; $n=6$ mice for SCCO28 lesions from experiment 1 (Exp.1) and 5 mice from experiment 2 . (A and B) Scale bars: $250 \mu \mathrm{m}$. Data are shown as mean \pm SEM. ${ }^{*} P<0.05 ;{ }^{* *} P<0.0005,1$-tailed paired $t$ test.

address this point, we resorted to an alternative skin tumorigenicity assay, based on intradermal injection of cells embedded in Matrigel.

Matrigel nodules form immediately after injection into animals and can be easily retrieved at any time. Parallel injections of SCC13 and SCCO28 cells with or without CSL gene silencing were used for these assays. Matrigel nodules retrieved at 1 week had sizes comparable to those of SCC cells with or without CSL silencing, with more detectable differences starting to occur at 2 weeks (Figure 8A). Histological features and inflammation, as detected by pan-keratin and CD45 staining, respectively, were comparable in all cases (Supplemental Figure 4, A and B). To assess intrinsic differences in gene expression, we resorted to laser capture microdissection (LCM) of pan-keratin-positive SCC cells, followed by RTqPCR analysis. Results showed significant upregulation of IL 6 and CCL2O, a leukocyte chemotactic cytokine under positive IL6 con- trol (50), in SCC13 and SCCO28 cells, with silenced CSL at both 1 and 2 weeks, with less consistent differences for 2 other cytokines, IL1-A and CXCL1 (Figure 8, B and C).

Similar intradermal tumorigenicity assays were performed with SCC13 cells with increased CSL expression via lentiviral vector infection. At 2 weeks after injection, Matrigel nodules harboring SCC cells with increased CSL expression exhibited a notably larger size than controls (Figure 8D), in agreement with previous results obtained by ear-injection assays. Histological features and inflammation were also comparable in all cases (Supplemental Figure 4, C and D). LCM and RT-qPCR analysis at both 1 and 2 weeks revealed significant reduction of IL6 and CCL2O expression in SCC cells with increased CSL, mirroring the inverse effects of CSL gene silencing (Figure 8E). Differences in expression of $I L 1-A$ and $C X C L 1$ were again less consistent (Figure 8E). 
A

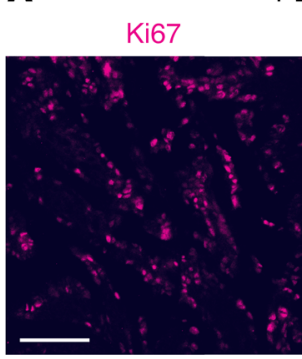

PLKO1 Ki67 Pan-keratin DAPI

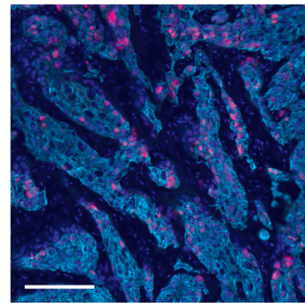

PLKO1

B

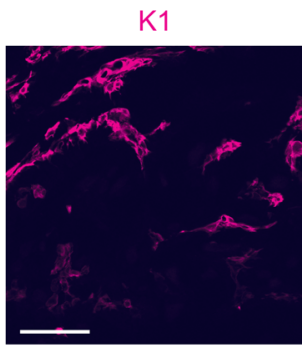

K1 Pan-keratin DAPI

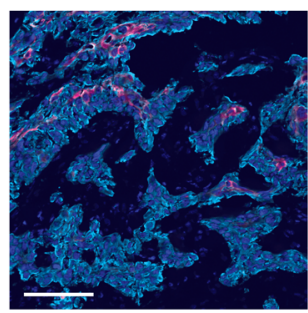

shCSL
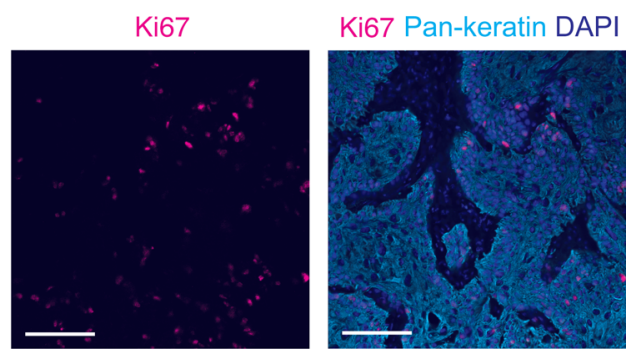

shCSL

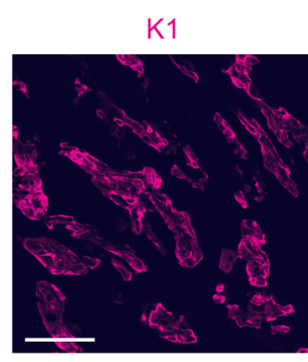

K1 Pan-keratin DAPI
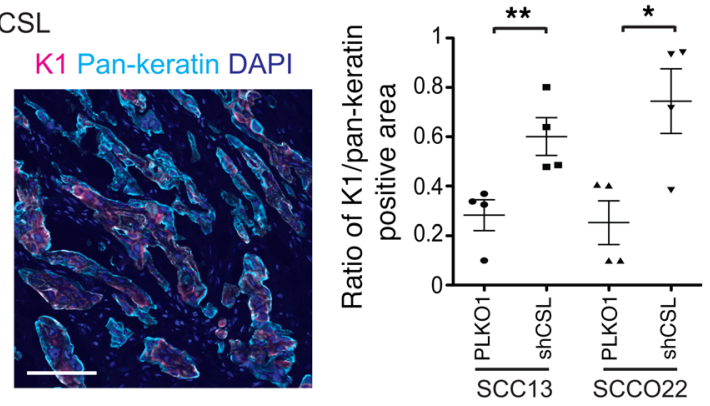

C

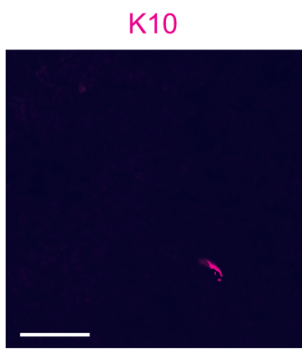

PLKO1

K10 Pan-keratin DAPI

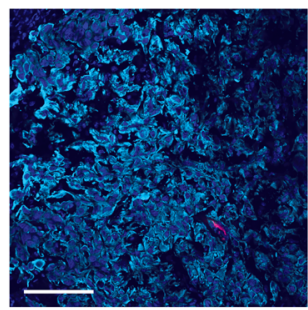

ShCSL

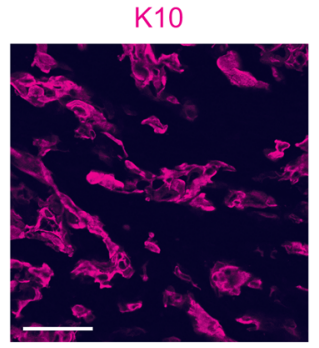

K10 Pan-keratin DAPI

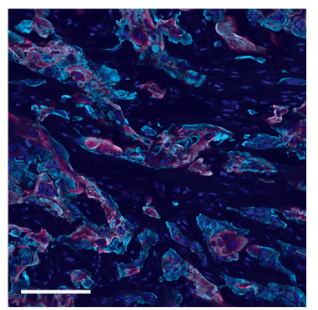

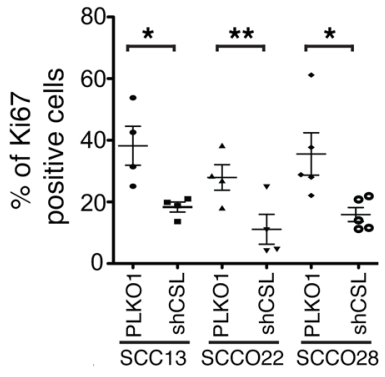

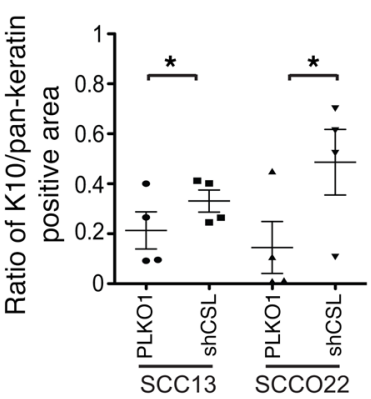

Figure 6. Decreased proliferation and increased differentiation in tumors formed by SCC cells with CSL gene silencing. (A) Double-immunofluorescence analysis of proliferation (Ki67) and epithelial (pan-keratin) markers in ear lesions formed by SCC13, SCCO22, and SCCO28 cells with CSL gene silencing. Shown are representative images as well as quantification of Ki67-positive nuclei in keratin-positive regions using Image) software. (B and C) Doubleimmunofluorescence analysis with antibodies against the K1 and K10 differentiation markers versus total keratins (pan-keratin) of ear lesions formed by SCC13 and SCCO22 cells with CSL gene silencing. Shown are representative images as well as quantification of K1 and K10 in pan-keratin-positive regions using ImageJ software. (A-C) Scale bars: $150 \mu \mathrm{m}$. Data are shown as mean \pm SEM. $n=4$ mice for SCC13 lesions; $n=4$ mice for SCCO22 lesions; $n=5$ mice for SCCO28 lesions. ${ }^{*} P<0.05 ;{ }^{* *} P<0.005,1$-tailed paired $t$ test.

KDM6B as direct target and mediator of CSL regulatory function in keratinocyte/SCC proliferation and tumorigenesis. For further mechanistic insights, we sought to identify direct CSL targets whose upregulation by CSL gene silencing could impinge on cell proliferation and tumor formation. Out of several hundreds of CSL target genes identified by ChIP-seq analysis (GEO GSE102761) (Supplemental Table 3), 68 were found by RNA-seq to be significantly upregulated by silencing of the gene in HKCs (Figure 9A). These coded for enzymes involved in signal transduction and metabolism, secreted factors, and cell surface and cytoskeletal proteins as well as transcription factors and chromatin modifiers (Figure 9B). Out of these genes, 13 were also upregulated by CSL silencing in SCC13 cells, including HES1, a "canonical" NOTCH/CSL target variously implicated in keratinocytes (4, 51-53), and KDM6B, a histone H3-K27 demethylase with contextdependent functions in cancer development (22) (Figure 9B). Direct ChIP assays on HKCs confirmed CSL binding to multiple sites of the KDM6B locus (Figure 9C) and to the HES1 promoter as a positive control (Figure 9D). Upregulation of the KDM6B gene by CSL gene silencing was confirmed by RT-qPCR in HKCs and SCC cells analyzed in this study, including SCCO28 cells (Figure 10, A and B). Upregulation of the KDM6B protein was also found by immunofluorescence analysis of HKCs and SCC cells with silenced CSL (Figure 10C). Even in vivo, in intradermal Matrigel assays described above, KDM6B expression was pronouncedly upregulated in SCC13 and SCCO28 cells with silenced CSL (Figure 10D). Conversely, KDM6B expression was found to be downregulated by LCM and RT-qPCR analysis of AK and SCC lesions versus flanking normal epidermis (Figure 10E).

To assess functional significance of KDM6B upregulation, HKCs were infected with retroviral vectors overexpressing KDM6B in WT versus demethylase-defective mutant forms (33) in parallel with empty vector control. Expression of functionally active $K D M 6 B$, but not the inactive form, was sufficient to 
Table 1. GO analysis of upregulated genes by RNA-seq analysis of HICs and SCC13 cells upon CSL gene silencing

Upregulated upon CSL silencing

GO term

60:0042981, regulation of apoptosis

C0:0043067, regulation of programmed cell death

G0:0001944, vasculature development

G0:0006464, protein modification process

G0:0009966, regulation of signal transduction

60:0022604, regulation of cell morphogenesis

G0:0007243, protein kinase cascade

G0:0007242, intracellular signaling cascade

G0:0007264, small GTPase-mediated signal transduction

G0:0008285, negative regulation of cell proliferation
HKC

$P$ value
$1.43 \times 10^{-06}$
$2.21 \times 10^{-06}$
$5.20 \times 10^{-05}$
$3.16 \times 10^{-04}$
$5.23 \times 10^{-04}$
0.001617
0.001776
0.002385
0.004627
0.016505

Adjusted $P$ value

$5.35 \times 10^{-04}$

$6.90 \times 10^{-04}$

0.010785

0.036405

0.056092

0.114317

0.120358

0.138661

0.195421

0.400453
SCC13

$\begin{array}{cc}P \text { value } & \text { Adjusted } P \text { value } \\ 4.18 \times 10^{-06} & 8.22 \times 10^{-04} \\ 5.99 \times 10^{-06} & 9.64 \times 10^{-04} \\ 3.88 \times 10^{-06} & 8.57 \times 10^{-04} \\ 0.010404 & 0.182356 \\ 3.48 \times 10^{-13} & 6.17 \times 10^{-10} \\ 3.75 \times 10^{-07} & 1.66 \times 10^{-04} \\ 2.74 \times 10^{-06} & 6.92 \times 10^{-04} \\ 1.33 \times 10^{-07} & 7.85 \times 10^{-05} \\ 0.006576 & 7.47 \times 10^{-04} \\ 0.017633 & 0.249064\end{array}$

reproduce the inhibitory effects of CSL silencing on cellular proliferation and colony formation in 3 different HKC strains (Figure 11, A and B). Conversely, silencing of KDM6B counteracted growth-inhibitory effects of CSL knockdown in SCC13 and, to a lesser extent, in SCCO22 cells (Figure 11C). Overall colonyforming capability was increased in cells with dual silencing of $C S L$ and KDM6B genes relative to cells with CSL silencing alone (Figure 11D), with particular increases in colonies of larger size, thought to originate from cells with greater proliferative capacity (45) (Figure 11E).

At the gene-expression level, counteracting effects were detected upon concomitant silencing of CSL and KDM6B on key cell-cycle and proinflammatory genes (such as CCNB1, CDK1, and $I L 6)$, with no effects on others (such as CCND1, CASP8, or BID) (Figure $11 \mathrm{~F}$ ), pointing to KDM6B as a main, but not the only, mediator of $C S L$-silencing effects on gene transcription.

A key question was to what extent $K D M 6 B$ upregulation is involved in the tumor suppression that CSL silencing exerts in vivo. Accordingly, 3 different SCC cell lines (SCC13, SCCO22, and SCCO28) with shRNA-mediated silencing of CSL individually and in combination with $K D M 6 B$ knockdown were injected into ears of immune-compromised mice. As shown in Figure 12A, the size of cancer lesions was dramatically enhanced by dual silencing of
CSL and KDM6B compared with CSL silencing alone, with significant increases in the fraction of cancer cells positive for proliferation marker Ki67 (Figure 12B). Enhanced tumor formation by dual $C S L$ and KDM6B gene silencing was accompanied by decreased expression of $I L 6$ and reduced recruitment of inflammatory cells, reversing the effects of CSL gene-silencing alone (Figure 12, C and D, to be compared with Supplemental Figure 3, A and B).

Thus, $K D M 6 B$ is a direct $C S L$-negative target with an inverse opposite role in control of HKC and SCC proliferative capacity, tumorigenesis, and tumor-associated inflammatory reaction.

\section{Discussion}

Proliferation and differentiation of squamous epithelial cells are tightly regulated through integrated signaling networks, with NOTCH activation as a critical player $(1,2)$. We show here that, besides its role as a mediator of NOTCH activation, CSL has another and opposite function in SCC development. In contrast to NOTCH, CSL levels are inversely correlated with keratinocyte differentiation and are already upregulated at premalignant stages of skin and oral SCC development. This is of functional significance, as increased CSL expression enhances keratinocyte and SCC proliferation potential and tumor formation, while CSL downmodulation suppresses them.

\section{Table 2. GO analysis of downregulated genes identified by RNA-seq analysis of HKC and SCC13 cells upon CSL gene silencing}

Downregulated upon CSL silencing

CO Term

G0:0007067, mitosis

G0:0000087, M phase of mitotic cell cycle

C0:0006260, DNA replication

CO:0006281, DNA repair

G0:0007051, spindle organization

G0:0000075, cell-cycle checkpoint

C0:0010564, regulation of cell-cycle process

60:0007346, regulation of mitotic cell cycle

G0:0051783, regulation of nuclear division

G0:0010824, regulation of centrosome duplication
HKC

$\begin{array}{cc}P \text { value } & \text { Adjusted } P \text { value } \\ 7.30 \times 10^{-58} & 3.65 \times 10^{-55} \\ 5.70 \times 10^{-57} & 2.14 \times 10^{-54} \\ 5.29 \times 10^{-48} & 1.59 \times 10^{-45} \\ 1.06 \times 10^{-38} & 2.65 \times 10^{-36} \\ 3.46 \times 10^{-24} & 7.41 \times 10^{-22} \\ 3.56 \times 10^{-17} & 6.67 \times 10^{-15} \\ 8.06 \times 10^{-11} & 8.06 \times 10^{-09} \\ 3.82 \times 10^{-10} & 2.86 \times 10^{-08} \\ 1.78 \times 10^{-04} & 0.006051 \\ 0.00451 & 0.093734\end{array}$

SCC13

$\begin{array}{cc}P \text { value } & \text { Adjusted } P \text { value } \\ 1.56 \times 10^{-11} & 3.81 \times 10^{-09} \\ 4.53 \times 10^{-12} & 1.39 \times 10^{-09} \\ 7.83 \times 10^{-11} & 1.37 \times 10^{-08} \\ 1.42 \times 10^{-05} & 0.001082 \\ 0.00207 & 0.100212 \\ 1.58 \times 10^{-05} & 0.001136 \\ 3.44 \times 10^{-05} & 0.002211 \\ 1.82 \times 10^{-04} & 0.011059 \\ 0.02541 & 0.418925 \\ 0.01231 & 0.308999\end{array}$




\section{Table 3. GSEA of differentially expressed genes in HKC and SCC13 cells upon CSL gene silencing}

Genes modulated upon CSL silencing

Cene sets

NGUYEN_NOTCH1_TARGETS_UP

GO_KERATINOCYTE_DIFFERENTIATION

TCFB_UPVI_UP

HINATA_NFKB_TARGETS_KERATINOCYTE_UP

HALLMARK_TNFA_SIGNALING_VIA_NFKB

HALLMARK INTERFERON_GAMMA RESPONSE

HALLMARK INFLAMMATORY_RESPONSE

HALLMARK_APOPTOSIS
HALLMARK_IL6_JAK_STAT3_SIGNALING

AS
-0.557
-0.373
0.693
0.709
0.738
0.81
0.688
0.705
0.548

HKC

FDR $q$ value

0.856

0

0.01

0

0

0

0.001

0.029

\begin{tabular}{cc}
\multicolumn{2}{c}{ SCC13 } \\
AS & FDR q value \\
-0.396 & 0.953 \\
-0.337 & 0.966 \\
0.7 & 0 \\
0.697 & 0.011 \\
0.721 & 0 \\
0.526 & 0.048 \\
0.565 & 0.014 \\
0.659 & 0.007 \\
0.515 & 0.065 \\
\hline
\end{tabular}

AS, association score.

While increased CSL expression enhanced the proliferative potential of primary HKCs and various SCC cell lines, its silencing exerted opposite effects. Growth-inhibitory effects of $C S L$ gene silencing can be explained by downregulation of positive regulators of the cell cycle, consistent with the thinner epidermis and reduced proliferation previously reported in mice with keratinocyte-specific deletion of the CSL gene (53). The further widespread alterations in the skin of these mice were attributed to skin barrier defects associated with strong induction of thymic stromal lym-

In Drosophila, it has long been known that the role of $\mathrm{Su}(\mathrm{H})$ (CSL homologue) as mediator of NOTCH activation cannot explain the different phenotypes resulting from loss of either gene (14-16). Increasing evidence indicates that, also in mammalian systems, CSL and NOTCH play only partially overlapping functions. In specific contexts, such as in conversion of stromal fibroblasts into cancer-associated fibroblasts (CAFs), loss or downmodulation of $C S L$, rather than recapitulating loss of NOTCH effects, can trigger the same response as NOTCH activation (40).

The possibility of distinct roles of CSL and NOTCH in cancer cells has been investigated to a very limited extent, with apparently conflicting results. A recent study focused on breast cancer and Burkitt lymphoma, in which NOTCH acts as an oncogene, pointed to an opposite tumor-suppressive role for CSL (54). Specifically, CSL silencing in MDA-MB-231 human breast cancer and DG75 Burkitt lymphoma cells enhanced tumor growth, with $c-M Y C$ - and NF-KB-dependent increases in cell survival. However, an opposite conclusion was suggested by other studies with cultured cancer cell lines, including one that was also with MDA-MB-231 cells (55-57). Thus, the possibility of distinct roles of NOTCH and CSL in specific cancer types needs further assessment in the context of their cells of origin and tumor microenvironment. The present work represents such an attempt, as it relates to primary keratinocytes and SCC.

We have found that in normal skin and oral epithelia, CSL expression is inversely related to differentiation. Complex posttranscriptional mechanisms are likely involved, at least in differentiating primary keratinocytes in which downmodulation of CSL protein levels was associated with increased (rather than decreased) mRNA expression. Perturbed differentiation is a key feature of squamous cancer development (1). Consistently, increased CSL levels were already found in premalignant skin and oral SCC lesions and SCC cells. While parallel increases of CSL mRNA and protein levels were detected in some SCC cells, in others, only the protein was upregulated. Posttranscriptional control of CSL expression and function is a complex and poorly understood topic currently under investigation. Here, we focused on the functional significance of changes in CSL levels in skin and head and neck epithelial cells. phopoietin (TSLP) cytokine production $(7,58)$. TSLP deregulation may be a peculiarity of the murine system, as it was not observed in human primary keratinocytes and SCC cell lines upon CSL gene silencing. Nevertheless, other cytokines and chemokines with potent proinflammatory function were instead consistently induced, particularly $I L 6$, a pleiotropic regulator of the immune and inflammatory systems (59) that has cell-autonomous growthinhibitory function $(59,60)$, and CCL2O, a leukocyte chemotactic factor under positive $I L 6$ control (50). Importantly, expression of these cytokines was enhanced in all SCC cells with CSL silencing and also in vivo, in an early assay of SCC development preceding differences in tumor size and inflammation. As such, expression of these cytokines is an intrinsic rather than a secondary event of decreased CSL expression in SCC cells. This can have a pleiotropy of functions at early versus late stages of tumor development, when substantially increased inflammatory reaction was found in association with tumor suppression. An increased immune-cell infiltration into tumors is a sign of a favorable response to checkpoint inhibitors (34), and it will be interesting to assess whether the regulatory network that we have uncovered correlates with immune infiltration in SCCs and/or their response to therapy.

Consequences of changes in CSL levels can be ascribed, in part, to direct negative control of the histone demethylase KDM6B gene, which impinges on genes with key cell-cycleregulatory and proinflammatory functions (23). This gene is essential for life after birth, as knockout mice are born smaller and die perinatally due to respiratory failure $(21,61,62)$. Previous studies have pointed to a potential tumor-suppressive role of $K D M 6 B$ in specific tissues (such as lung, colon, and pancreas) (30-32, 63), while in others (such as blood, breast, and brain) it may play an opposite tumor-promoting function (24-26). This demethylase is an important player at the intersection between cellular senescence and cancer $(22,29)$, and it also positively regulates epidermal differentiation (33). Interestingly, the KDM6B gene is located on chromosome 17 in close vicinity to the $p 53$ tumor-suppressor gene, with allelic loss at this position occurring in a variety of cancers, including SCCs $(1,22,64-66)$.

A number of direct CSL target genes commonly upregulated by its silencing in HKCs and SCC13 cells could be involved in the 

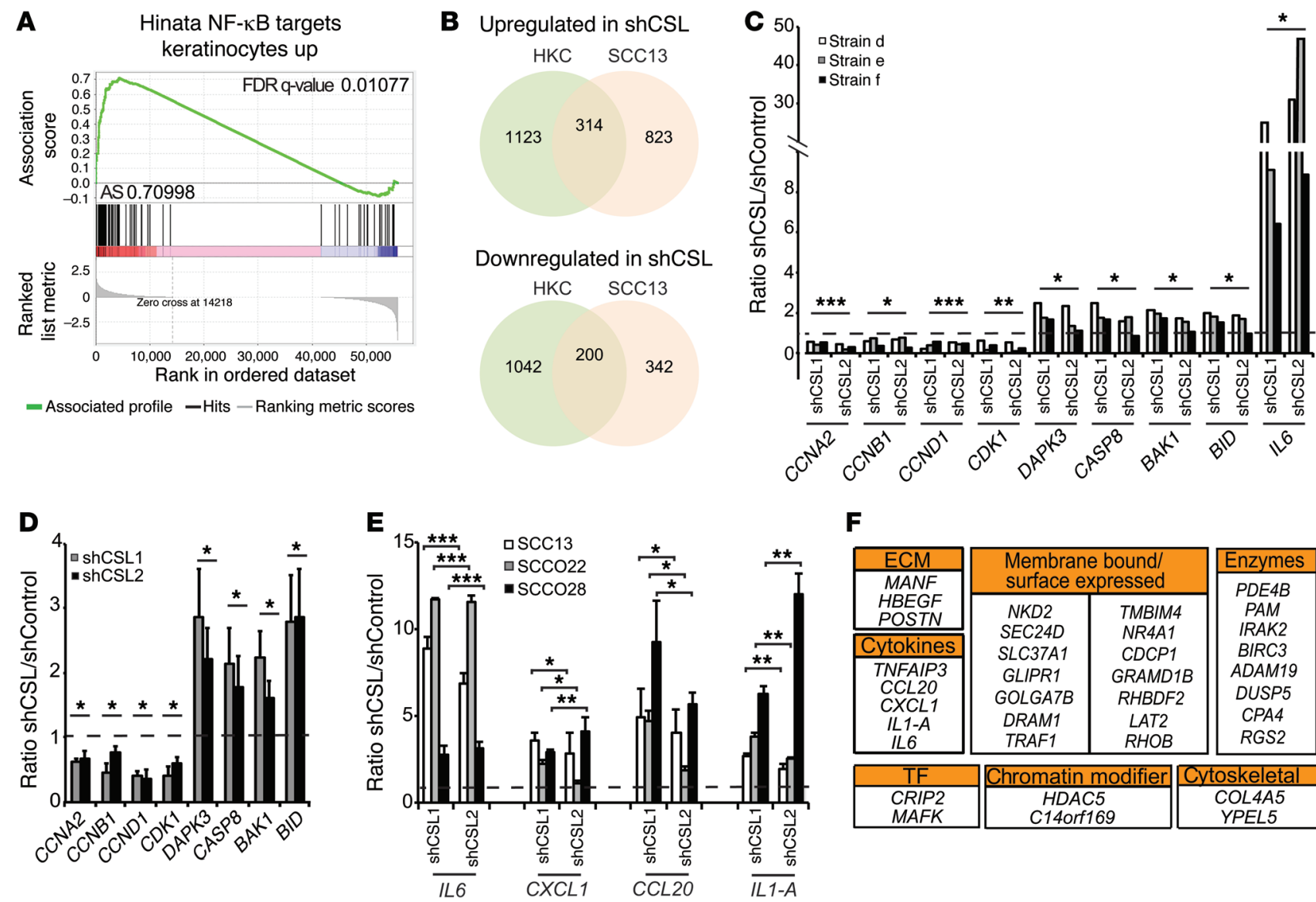

F

\begin{tabular}{|c|c|c|c|}
\hline ECM & \multirow{2}{*}{\multicolumn{2}{|c|}{$\begin{array}{l}\text { Membrane bound/ } \\
\text { surface expressed }\end{array}$}} & \multirow{3}{*}{\begin{tabular}{|c|} 
Enzymes \\
PDE4B \\
$P A M$ \\
IRAK2
\end{tabular}} \\
\hline MANF & & & \\
\hline POSTN & $N K D 2$ & TMBIM4 & \\
\hline Cytokines & SLC $37 A 1$ & CDCP1 & $B I R C$ \\
\hline TNFAIP3 & GLIPR1 & GRAMD1B & ADAM19 \\
\hline CXCL1 & GOLGA7B & RHBDF2 & DUSP5 \\
\hline $\begin{array}{l}\operatorname{LL1-A} \\
\| L 6\end{array}$ & DRA & & \\
\hline & & & \\
\hline 11 & Shromatin & difier & \\
\hline $\begin{array}{l}\text { CRIP2 } \\
\text { MAFK }\end{array}$ & $\begin{array}{r}H D \\
C 140 \\
\end{array}$ & & $\begin{array}{l}\text { COL4A5 } \\
\text { YPEL5 }\end{array}$ \\
\hline
\end{tabular}

Figure 7. RNA-seq analysis of normal HKCs and SCC cells upon CSL silencing. (A) Representative plot of gene distribution in HKCs upon CSL silencing against an experimental gene set of NF- $\mathrm{KB}$ targets from CSEA shown in Table 3. (B) Venn diagram illustrating number of up- and downregulated genes overlapping in RNA-seq profiles of HKCs and SCC13 cells upon CSL silencing. (C) Three independent HKC strains infected with 2 shRNA lentiviruses against CSL were analyzed by RT-qPCR for indicated genes with 36B4 for normalization. $n=3$ HKC strains. (D) SCC13 cells infected with 2 shRNA lentiviruses against CSL were analyzed by RT-qPCR for indicated genes using 36B4 for normalization. $n=3$ independent experiments. Data are shown as mean \pm SEM. (E) Panel of SCC cell lines infected with 2 shRNA lentiviruses against CSL were analyzed by RT-qPCR for indicated genes using $36 B 4$ for normalization. $n=3$ independent experiments. Data are shown as mean \pm SEM. (F) List of genes upregulated by RNA-seq analysis in SCCO28 cells upon CSL gene silencing divided by biological function. ECM, extracellular matrix; TF, transcription factors. (C-E) ${ }^{*} P<0.05 ;{ }^{* *} P<0.005$; ${ }^{* *} P<0.0005$, 2-tailed 1-sample $t$ test followed by Benjamini-Hochberg correction.

observed biological effects. Among these, KDM6B was the only epigenetic regulator with potential global impact on gene transcription. $K D M 6 B$ upregulation is of functional relevance, as its silencing counteracted growth-inhibitory effects of CSL knockdown in SCC cells, both in vitro and in vivo. Mechanistically, this could be explained by the previously reported role of KDM6B in direct transcriptional control of cell-cycle-inhibitory genes, such as $\operatorname{CDKN2A}(22,29)$, differentiation genes, such as $K 1(33)$, and cytokines with proinflammatory functions, specifically IL6 (35, 37). Consistent with the latter mechanism of action, we found that upregulation of IL6 expression by CSL silencing was suppressed by concomitant $K D M 6 B$ knockdown, which parallels its in vivo effects in rescuing tumor formation.

There are several possible mechanisms by which $K D M 6 B$ can regulate the above genes. $K D M 6 B$ can directly associate with key transcription factors, such as $p 53(67,68), \mathrm{p} 65$, and p50 NF-KB subunits $(37)$ and STAT $(69,70)$ and SMAD (71) family members, enhancing their functions. In turn, a reciprocal mode of regulation of KDM6B by NF-KB and STATs observed in other cell types (36, $38,70,72,73)$ may also apply to squamous cancer, which is often associated with and can be induced by inflammation (74).

Targeting epigenetic regulators is emerging as promising cancer therapy. Pharmacological inhibitors of KDM6B (such as GSKJ4) (75) were shown to inhibit growth of different cancer cell lines, such as T-ALL and glioma $(20,21,76,77)$, in which KDM6B plays an oncogenic role (22). While use of these compounds would obviously be contraindicated for squamous cancer, other drugs enhancing KDM6B function or inhibiting epigenetic modifying enzymes with an opposite role could be of benefit and open novel windows of opportunity for both prevention and treatment of this disease.

More generally, our findings could lead to valuable prognostic tools to be used, in combination with other markers, in the clinic. This is suggested by a significant association between increased CSL expression and high-grade SCCs in tissue microarrays (Figure $1 \mathrm{E}$ ) and detectable differences in expression of this protein in normal keratinocytes versus SCC cells processed as for analysis 

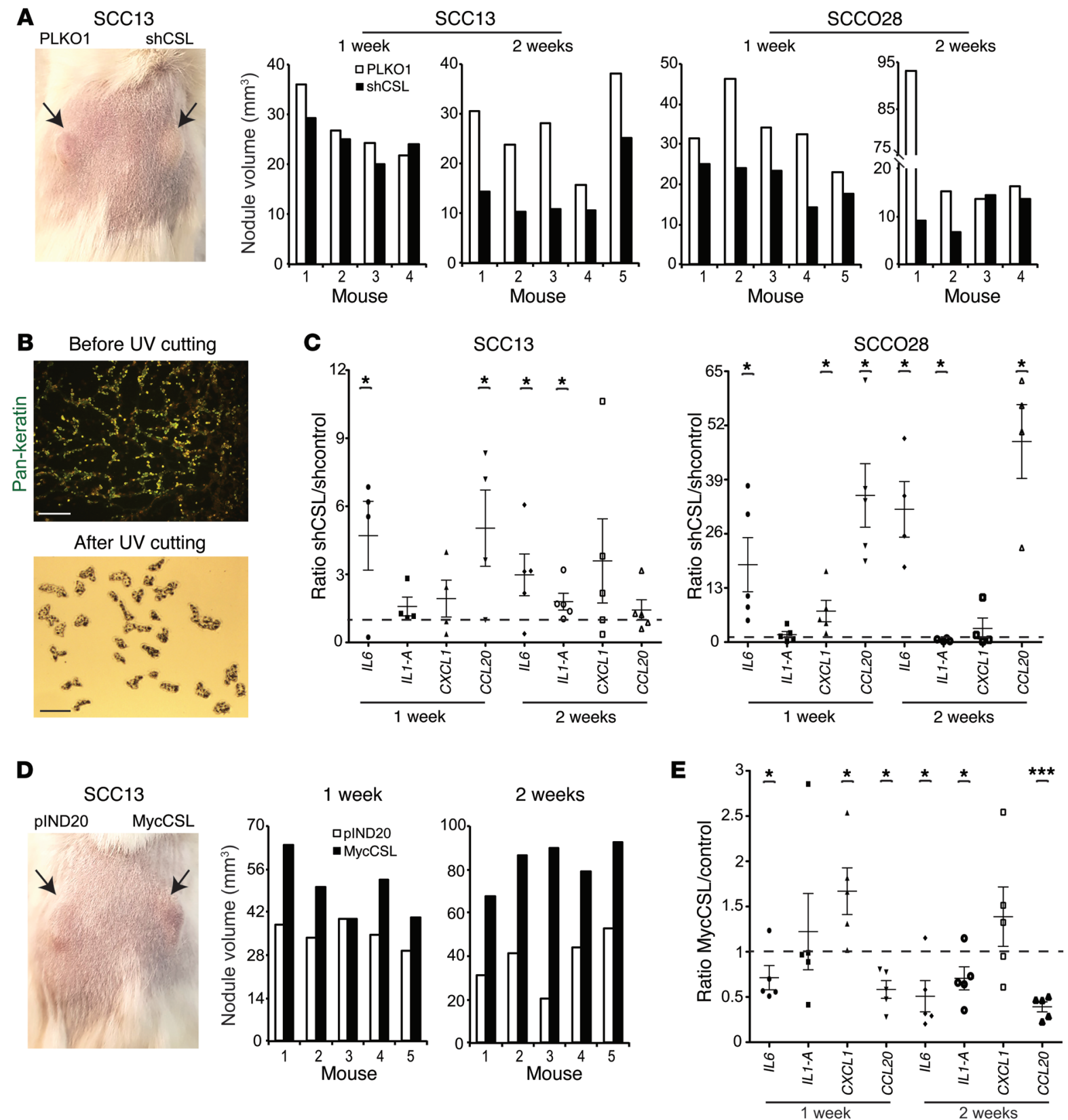

Figure 8. Effect of CSL modulation on inflammatory cytokines in vivo. (A) SCC13 and SCCO28 cells infected with CSL-silencing lentivirus versus empty vector control were injected into backs of NOD/SCID mice. Shown are a representative image of 1 mouse (left panel) and nodule volumes at weeks 1 and 2 after injection (right panel). (B) Representative images of nodule sections processed for fluorescence-guided LCM utilizing anti-pan-keratin-Alexa Fluor 488-conjugated antibody. Shown is a stained section before UV cutting (upper panel) and captured material on an LCM cap after UV cutting (lower panel). Scale bars: $150 \mu \mathrm{m}$ (upper panel); $500 \mu \mathrm{m}$ (lower panel). (C) RNA samples from LCM-captured material were examined by RT-qPCR for cytokine marker expression. $n=4$ mice for SCC13 at week 1; $n=5$ mice for SCC13 at week 2; $n=5$ mice for SCCO28 at week 1; $n=4$ mice for SCCO28 at week 2 . (D) SCC13 cells stably infected with lentiviral vector for doxycycline-inducible Myc-tagged CSL or empty vector control (pIND20) were injected into backs of NOD/SCID mice. Shown is a representative image of 1 mouse (left panel) and nodule volumes at weeks 1 and 2 after injection (right panel). (E) RNA samples from LCM-captured material were examined by RT-qPCR for cytokine marker expression. $n=5$ mice at week $1 ; n=5$ mice at week 2 . (C and E) Data are shown as mean \pm SEM. ${ }^{*} P<0.1 ;{ }^{* *} P<0.001,1$-tailed 1-sample $t$ test followed by Benjamini-Hochberg correction.

of cytological specimens. Similar assessment of KDM6B expression in clinical settings should also be possible with development of adequate antibodies. These are worthwhile goals, as there is an unmet need for biomarkers that help identify precancerous and cancerous squamous cancer lesions and predict their response to novel modalities of treatment.

\section{Methods}

Cells and human samples. Normal epithelial cells and SCC cells were cultured as previously described $(12,78)$. Oral SCC cells (SCCO11, SCCO13, SCCO22, and SCCO28) were provided by James Rocco (Massachusetts General Hospital, Boston, Massachusetts, USA). Oral SCC cells (Cal27, Cal33, and FaDu) were provided by Genrich Tolstonog 
A

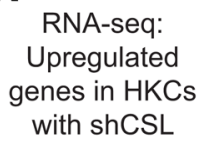

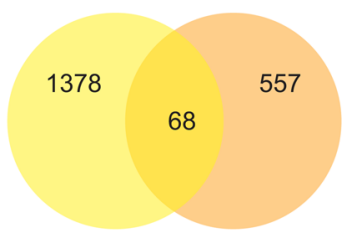

ChIP-seq

direct targets of

CSL in HKCs

B

\begin{tabular}{|c|c|c|c|}
\hline \multicolumn{2}{|c|}{ Enzymes } & \multirow{2}{*}{\multicolumn{2}{|c|}{$\begin{array}{l}\text { Membrane bound/ } \\
\text { surface expressed }\end{array}$}} \\
\hline KMO & TRIM5 & & \\
\hline PTK2 & RNF149 & SCARB2 & OCLN \\
\hline EXD3 & SPTLC2 & CNTNAP2 & TACSTD2 \\
\hline AKAP13 & PTPN14 & SNN & CLCN4 \\
\hline RND3 & DYRK2 & ANO6 & TMCC1 \\
\hline TRIM22 & PLEKHG1 & KCNK1 & DCBLD1 \\
\hline SCD5 & CCRN4L & NAPG & RALGPS1 \\
\hline HM13 & GNAI2 & SEC61A1 & DSP \\
\hline PIK3R3 & SPSB1 & SNX25 & PVRL4 \\
\hline Secreted factors & Cytoskeletal & FAT2 & RAB11FIP4 \\
\hline SPARC & MPRIP & SUSD1 & MAGT1 \\
\hline SLIT3 & TPM1 & NOTCH2 & RHOV \\
\hline CCL20 & MFAP2 & $\begin{array}{l}\text { ROBO2 } \\
\text { ROB }\end{array}$ & CEACAM19 \\
\hline TNFSF10 & ENC1 & $\begin{array}{l}\text { PLXDC2 } \\
\text { NRXN3 }\end{array}$ & VPS18 \\
\hline FGF1 & SHANK2 & & \\
\hline FRAS1 & Chromatin & TF act & /binding \\
\hline Pseudogene & modifiers & GAS7 & CASZ1 \\
\hline TMSB4X & NCOA2 & $\begin{array}{l}\text { HSF2BP } \\
\text { HES1 }\end{array}$ & $\begin{array}{l}\text { HIVEP2 } \\
\text { SP110 }\end{array}$ \\
\hline PLEKHA8P1 & KDM6B & ZNF469 & \\
\hline
\end{tabular}

C

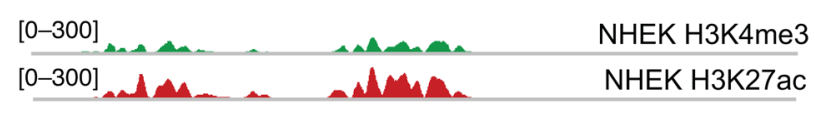

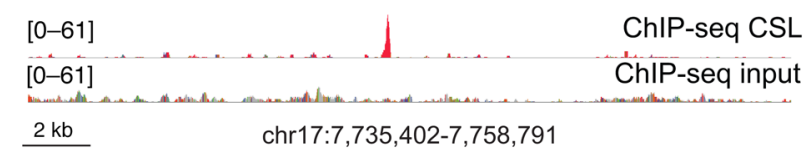

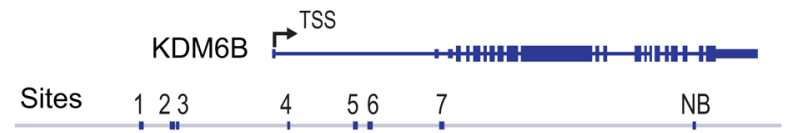

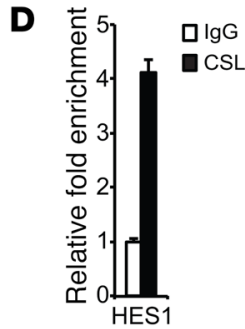

Figure 9. KDM6B as a direct target of CSL. (A) Venn diagram illustrating overlap between number of genes bound by CSL (ChIP-seq profile) and those upregulated by silencing of the gene (RNA-seq profile) in HKCs. (B) List of overlapping genes grouped by biological function. Highlighted are direct CSL target genes commonly upregulated by CSL gene silencing in HKCs and SCC13 cells. (C) Upper panel: graphic illustration of the position of CSL-binding peaks revealed by Chip-seq analysis for the KDM6B gene, utilizing ENCODE information for promoter and enhancer localization, as indicated by islands of histone H3 modifications (K4me3 and K27Ac) along with position of the transcription start site (TSS) (81) and coding exons (boxes). Also indicated are positions of sites tested by direct ChIP assays. Lower panel: ChIP assays for independent confirmation of Chip-seq results of CSL binding to the indicated sites within the KDM6B genomic locus. HKCs were processed for ChIP assays with anti-CSL antibodies versus nonimmune IgG controls, using tagmentation for signal amplification (see Methods). Shown are CSL fold enrichments relative to nonimmune controls (NB, nonbinding). (D) The same ChIP samples as in C were tested for CSL fold enrichment of predicted segments of the HES1 gene-promoter region as positive controls for the assay.

(CHUV). Skin SCC cells (SCC12 and SCC13) were provided by James Rheinwald (Brigham and Women's Hospital, Boston, Massachusetts, USA). Lung SCC cells (H520, H2170, HCC95, SW900, and SK-MES-1) were provided by Peter Hammerman (Dana-Farber Cancer Institute, Boston, Massachusetts, USA). HBECs were purchased from ATCC. HKC cells were prepared from discarded human foreskin samples from circumcision at the Department of Pediatrics (CHUV). In brief, skin tissue was immersed in $1 \%$ Dispase II (Roche) overnight at $4^{\circ} \mathrm{C}$, and the epidermis compartment was separated from the dermis. Then, epidermis was minced and digested by $0.25 \%$ Trypsin-EDTA (Gibco, Thermo Fisher Scientific) for 1 hour at $4^{\circ} \mathrm{C}$ with gentle agitation, followed by filtering through $40 \mu \mathrm{m}$ mesh. After enzymatic digestion was terminated by adding a volume of serum-containing media that was equal to the volume of the reaction, cell pellets were collected by centrifugation, plated, and cultured as previously described (41). Excised human AK samples not used for histological analysis were provided by Renato Panizzon, and human SCC samples were provided by Massimo Bongiovanni. Human SCC tissue arrays were purchased from US Biomax (SK2081 and OR802).

Cell manipulations and plasmid constructs. Conditions for culturing cells and viral infections were as previously reported (12, 40, 78). Briefly, HEK293 cells were cotransfected with viral packaging plas- mids and our plasmid of interest using Jetpei (Polyplus) transfection reagent for 6 hours, followed by virus collection after 48 hours. HKCs and SCC cells were infected for 1 hour and 6 hours, respectively, with high-titer lenti- and retroviruses sufficient to infect the majority of cells. Two days after infection, cells were selected with $1 \mu \mathrm{g} / \mathrm{ml}$ of puromycin for 3 days for shCSL and MSCV-KDM6B, with $500 \mu \mathrm{g} / \mathrm{ml}$ of G418 for 4 days for pmx-mycCSL and pIND20-mycCSL, and with $10 \mu \mathrm{g} / \mathrm{ml}$ of blasticidin for 4 days for shKDM6B.

Lentiviral vectors encoding shRNA against KDM6B were purchased from OriGene (HC141378B and HC141378C). Retroviral vectors encoding WT and mutant KDM6B (MSCV-KDM6B) (Addgene plasmid nos. 21212 and 21214) were a gift from Paul Khavari (Stanford University, Stanford, California, USA). Plasmids encoding for shCSL, pMXs-myc-CSL, and pINDUCER-20-myc-CSL have been previously described (40).

Cell assays. For proliferation assays, cell viability was assessed using the CellTiter-Glo Kit (Promega) by luminescence intensity determination over time. In brief, cells were plated onto 96-well plates (1,000 cells/well; triplicate wells/condition) 5 days after infection for shCSL and MSCV-KDM6B, 6 days after infection for pMXs-myc-CSL and shKDM6B, and 2 days after doxycycline treatment $(500 \mathrm{ng} / \mathrm{ml})$ for pIND20-mycCSL. Equal volumes of CellTiter-Glo reagent (50 $\mu$ l) and 

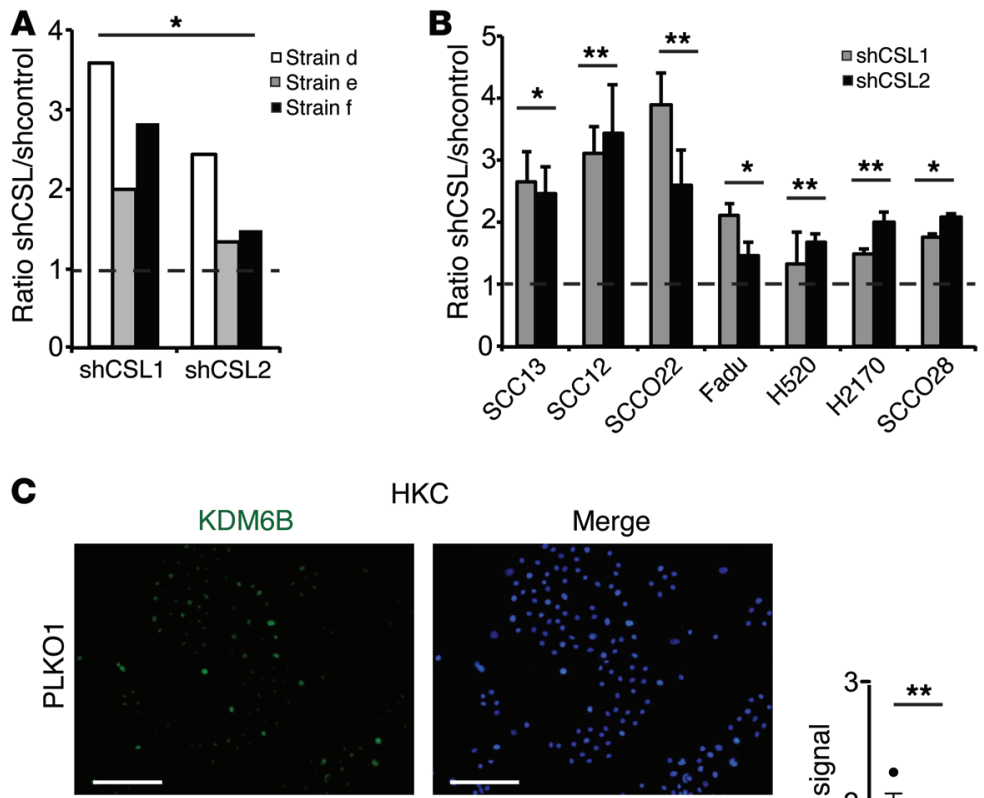

HKC
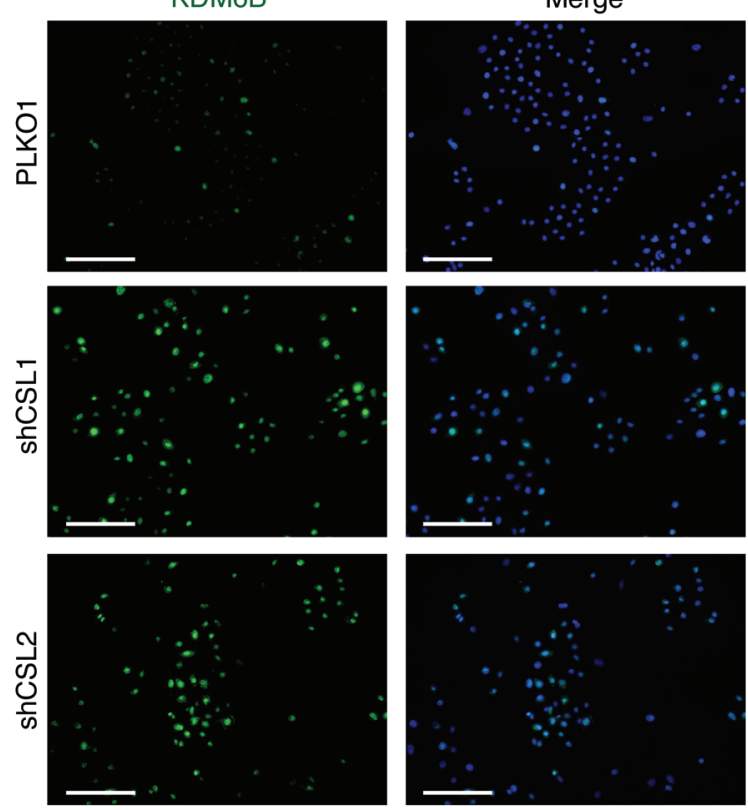

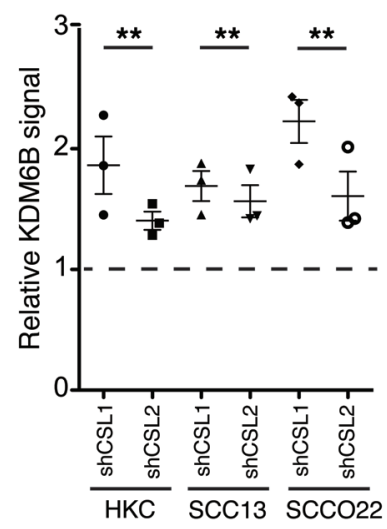

Figure 10. KDM6B expression increases in HICs and SCC cells with CSL gene silencing and decreases in neoplastic lesions. (A) Three independent HKC strains infected with 2 shRNA lentiviruses against CSL were analyzed by RT-qPCR for KDM6B mRNA expression with 36B4 for normalization; 2-tailed 1-sample $t$ test. $n=3$ HKC strains. (B) Indicated SCC cell lines infected with 2 shRNA lentiviruses against CSL were analyzed by RT-qPCR for KDM6B mRNA expression with 36B4 for normalization. Data are shown as mean \pm SEM, 2-tailed 1-sample $t$ test. $n=3$ independent experiments. (C) Immunofluorescence analysis of KDM6B expression in HKCs and SCC13 and SCCO22 cells with CSL gene silencing, with DAPI staining for cell identification. Shown are representative images of HKC staining (left panel) and quantification of fluorescence signal using Imagej software from all tested cell lines (right panel). Data are shown as mean \pm SEM, 2-tailed 1-sample $t$ test. Scale bars: $150 \mu \mathrm{m}$. (D) The same RNA samples as in Figure 8C were examined by RT-qPCR for KDM6B expression. Data are shown as mean \pm SEM, 1-tailed 1-sample $t$ test. $n=4$ mice for SCC13 at week $1 ; n=5$ mice for SCC13 at week 2 ; $n=5$ mice for SCCO28 at week $1 ; n=4$ mice for SCCO28 at week 2. (E) LCM-obtained epidermis from AK and skin SCC lesions versus flanking normal skin were analyzed by RT-qPCR for KDM6B expression. P, patients. $n=6 \mathrm{AK}$ regions; $n=6$ normal regions; $n=2$ SCC regions; $n=2$ normal regions. (A-E) ${ }^{*} P<0.05$; ${ }^{*} P<$ $0.005 ;{ }^{* *} P<0.0005,2$-tailed paired $t$ test.
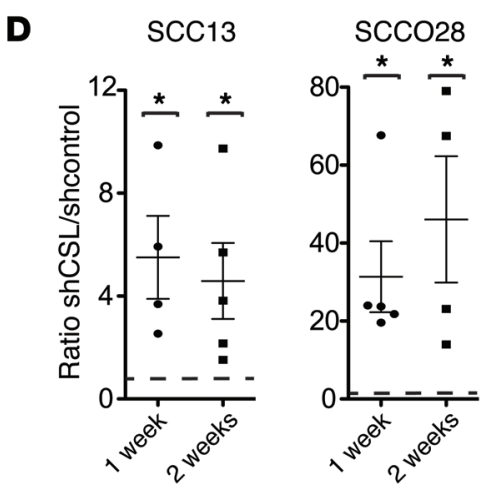

\section{E}

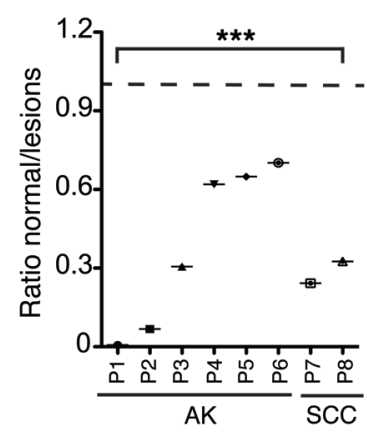

cell culture medium $(50 \mu \mathrm{l})$ were added to cells, followed by induced cell lysis on an orbital shaker for 3 minutes. The plate was incubated at room temperature (RT) for 10 minutes, after which luminescence was recorded using the Molecular Device Spectra Max Fluorometer.

For clonogenicity assays, cells were plated on $60 \mathrm{~mm}$ dishes (1,000 cells/well; triplicate wells/condition) and cultured for 10 days. Colonies were fixed with $4 \%$ formaldehyde and stained with $1 \%$ crystal violet.

For spheroid assays, cells were plated onto 8-well chamber slides coated with Matrigel (BD Biosciences). In brief, 8-well chambers were coated with $50 \mu$ l Matrigel per well and incubated for 30 minutes to polymerize. Cells were brought into suspension in normal culture medium plus 1\% Matrigel and plated for 8 days onto a precoated chamber.

For EdU assays, DNA synthesis was assessed using the Click-iT Plus EdU Imaging Kit (Life Technologies). Cells were incubated for 6 hours with $10 \mu \mathrm{M}$ EdU-labeling solution in prewarmed tissue culture medium. Cells were then fixed with $4 \%$ formaldehyde, followed by permeabilization with $0.5 \%$ Triton-X 100 . After permeabilization, cells were incubated with Click-iT Plus Reaction Cocktail for 30 minutes at RT, followed by counterstaining with DAPI.

For apoptosis assays, dead and preapoptotic cells were assessed using the Aqua Vivid Kit (Invitrogen) and the Annexin Kit (BD Biosci- 
A
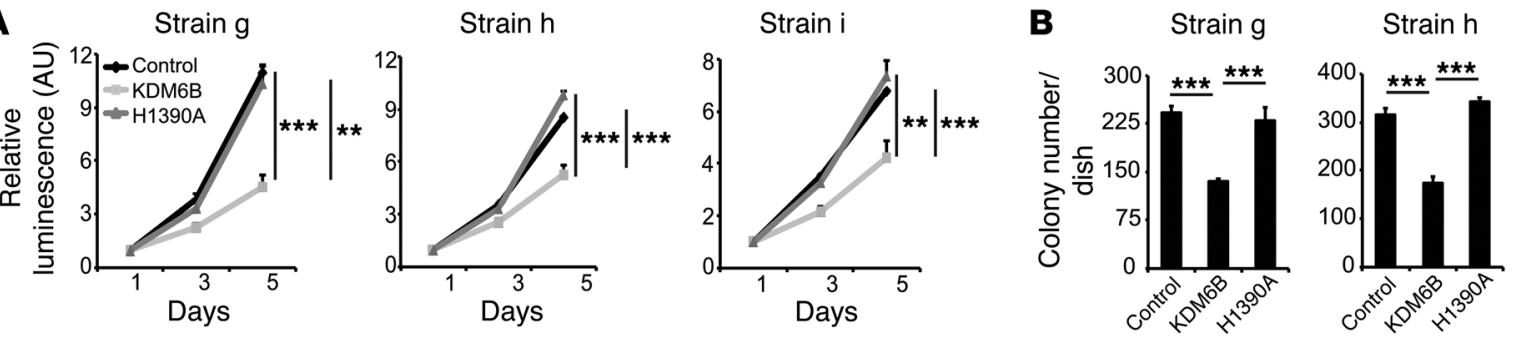

Strain $\mathrm{i}$
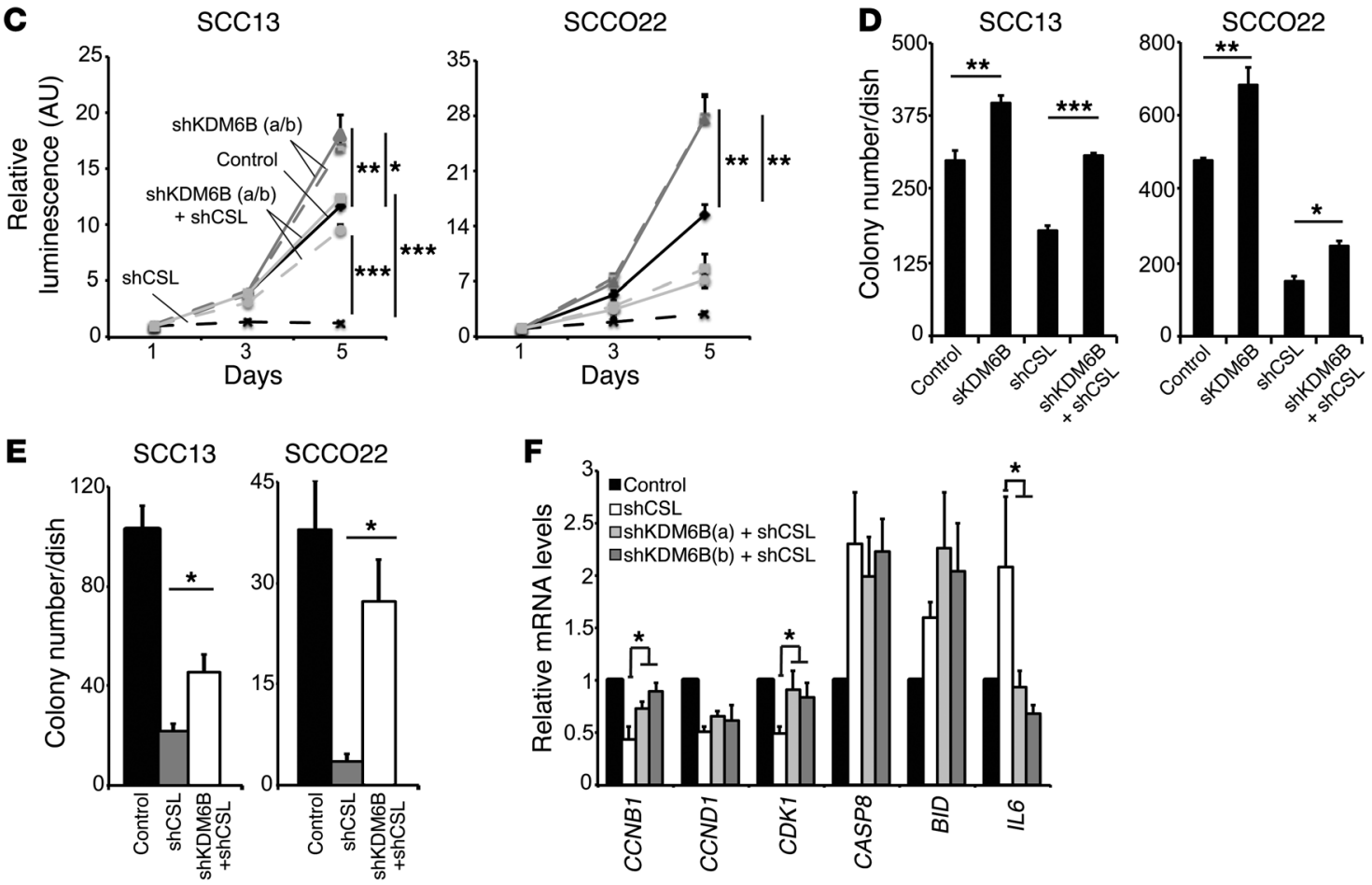

Figure 11. KDM6B as a downstream CSL target counteracting its growth-enhancing modulatory function. (A) Three independent strains of HKCs stably infected with retroviral viruses expressing KDM6B, KDM6B demethylase-defective point mutant (H1390A), and empty vector control were tested for cell metabolic activity assays over the indicated days. Data are shown as mean \pm SD. $n=3$ biological replicates/strain. (B) The same HKC strains as in $\mathbf{A}$ were plated at a limited density, and colony formation was measured. Data are shown as mean \pm SD. $n=3$ biological replicates/strain. (C) SCC13 and SCCO22 cells were infected with shRNA lentivirus against KDM6B or empty control, followed by infection with either control or CSL-silencing lentiviruses. Cells were tested for cell metabolic activity assays over the indicated days. Data are shown as mean \pm SEM. $n=3$ independent experiments. (D) The same cells as in $\mathbf{C}$ were plated at a limited density, and colony formation was measured. Data are shown as mean \pm SEM. $n=3$ independent experiments. (E) The same colony dishes as in $\mathbf{D}$ were assessed for the number of large colonies. (F) The same SCC13 cells as in $\mathbf{C}$ were analyzed by RT-qPCR for the indicated genes using 36B4 for normalization. Data are shown as mean \pm SEM. Two-tailed unpaired $t$ test, followed by Benjamini-Hochberg correction. $n=3$ independent experiments. $(\mathbf{A}-\mathbf{E}){ }^{*} P<0.1 ;{ }^{* *} P<0.01 ;{ }^{* *} P<0.001,1$-way ANOVA with Tukey's test.

ences), respectively. In brief, cells were harvested and incubated for 30 minutes on ice using the Aqua Dead Cell Stain Kit (Thermo Fisher Scientific). Cells were then washed with annexin-binding buffer, followed by incubation for 15 minutes at RT with annexin-Cy5 dye for staining of preapoptotic cells. Following annexin incubation, cell were fixed with $4 \%$ formaldehyde and counterstained with DAPI.

For gene expression, cells were collected for RNA followed by RTqPCR analysis. Total RNA ( $1 \mu \mathrm{g})$ was reverse-transcribed into cDNA using RevertAid H Minus Reverse Transcriptase (Thermo Scientific). Real-time qPCR was then done using SYBR Fast qPCR Master Mix (Kapa Biosystems) in Light Cycler 480 (Roche) according to the manufacturer's protocols. All RNA samples were analyzed in triplicate with gene-specific primers and 36B4 for normalization. The list of primers is provided in Supplemental Table 4.
Tumorigenesis experiments. Intradermal ear-injection assays were carried out in 6- to 8-week-old NOD/SCID mice with IL-2 receptor $\gamma$ chain null mutation (University of Lausanne). For in vivo silencing assays, SCC cells were infected with either control or shCSL viruses for 5 days and injected in equal number $\left(2 \times 10^{5}\right.$ cells per injection) into left and right ears of each mouse. For in vivo dual silencing assays, SCC cells were first infected with either control or ShKDM6B retroviruses for 6 days, followed by infection with shCSL lentiviruses for 5 days before injection into mouse ears $\left(2 \times 10^{5}\right.$ cells per injection). For in vivo overexpression assays, SCC cells were infected with either control or pIND20-mycCSL viruses and injected in equal number $\left(2 \times 10^{5}\right.$ cells per injection) into left and right ears of each mouse. Doxycycline $(1 \mathrm{mg} / \mathrm{ml})$ was freshly added to drinking water (containing $5 \%$ sucrose) every other day. In all cases, cells were concentrated 
A
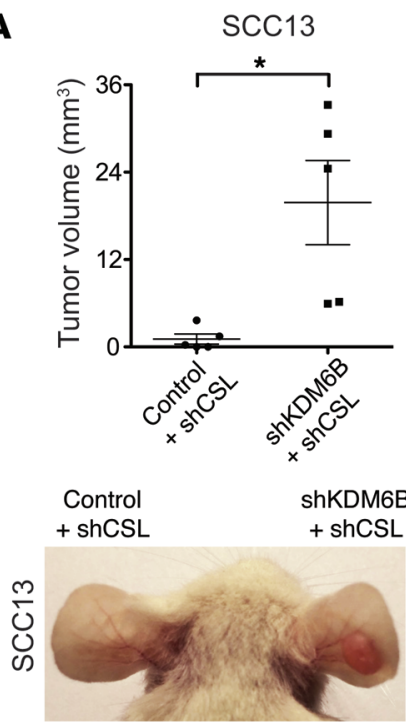
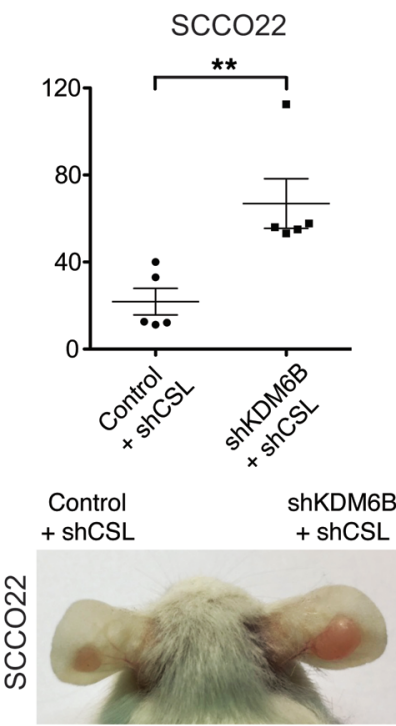
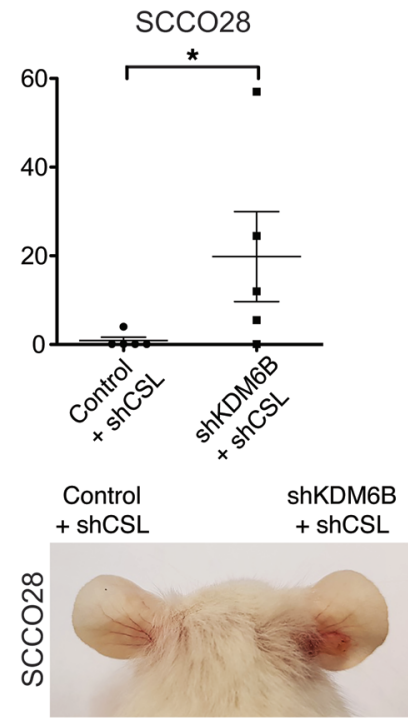

shKDM6B+ shCSL

B Control + shCSL

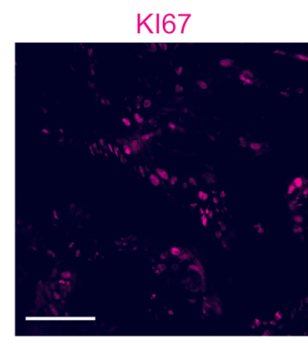
KI67 Pan-keratin DAPI
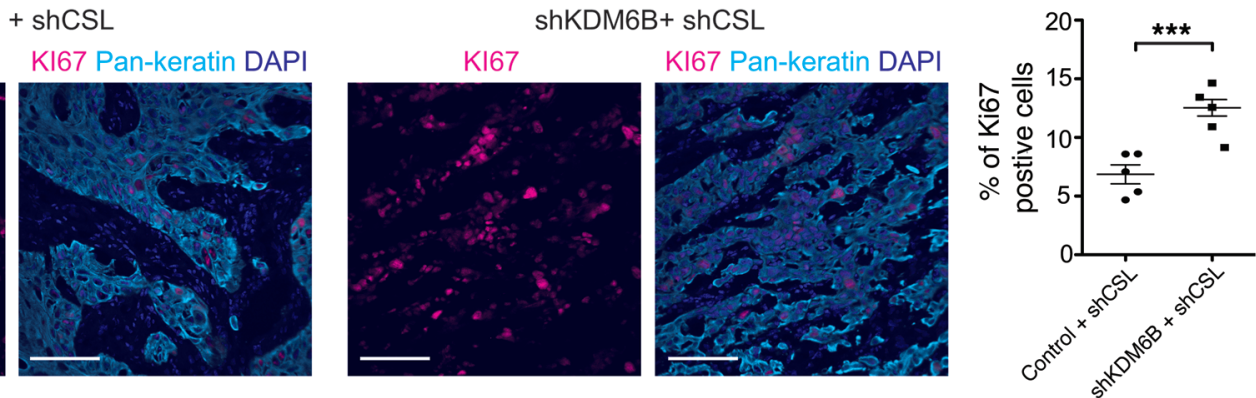

C IL-6

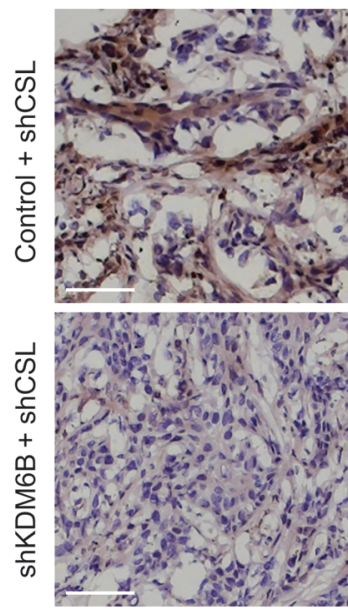

D

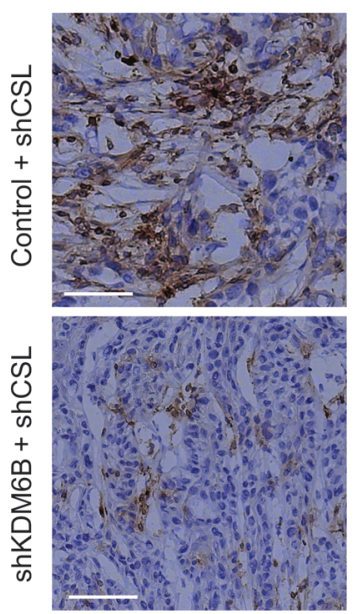

Control + shCSL

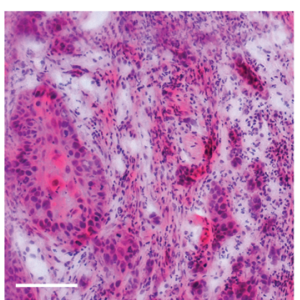

shKDM6B+ shCSL

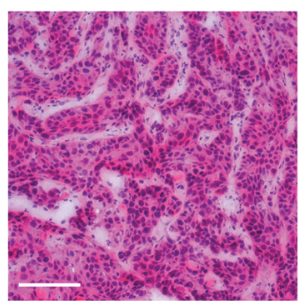

Figure 12. KDM6B as a downstream CSL target mediating its tumor-suppressive function. (A) SCC13, SCCO22, and SCCO28 cells freshly infected with shRNA lentiviruses against CSL or KDM6B individually or in combination, followed by parallel injection into ears of NOD/SCID mice. Shown are tumor volumes at day of sacrifice (upper panel) with representative images for 1 pair of mouse ears (lower panel) and corresponding H\&E staining of SCCO22 lesions (right panel). $n=5$ mice for SCC13 lesions; $n=5$ mice for SCCO22 lesions; $n=5$ mice for SCCO28 lesions. Scale bars: $250 \mu \mathrm{m}$. (B) Double-immunofluorescence analysis of proliferation (Ki67) and epithelial (pan-keratin) markers in ear lesions formed by SCCO22 cells. Shown are representative images as well as quantification of Ki67-positive nuclei in keratin-positive regions using Image) software. $n=5$ mice. Scale bars: $150 \mu \mathrm{m}$. (C and D) Immunohistochemical analysis of ear lesions formed by SCCO22 cells with antibodies against IL-6 and CD45. Shown are representative images as well as quantification of positive regions using ImageJ software. $n=5$ mice. Scale bars: $250 \mu \mathrm{m}$. (A-D) Data are shown as mean $\pm \mathrm{SEM}$. ${ }^{*} P<0.05 ;{ }^{* *} P<0.005$; ${ }^{* * *} P<0.0005,1$-tailed paired $t$ test. 
by centrifugation and resuspended in $3 \mu \mathrm{l}$ of medium per ear injection using a 33-gauge microsyringe (Hamilton). Mice were sacrificed for tissue analysis 5 to 8 weeks later, after the injection.

Intradermal back-injection assays were carried out in 6- to 8 -week-old NOD/SCID mice with IL-2 receptor $\gamma$ chain null mutation (University of Lausanne). SCC cells were infected with the virus of interest, brought into suspension, and admixed with $70 \mu \mathrm{l}$ Matrigel (BD Biosciences), followed by injection of equal numbers $\left(10^{6}\right.$ cells per injection) into left and right sides of the mouse back. Mice were sacrificed for tissue analysis 1 week and 2 weeks after injection.

Tumor size was measured using a digital caliper, and volume was calculated using the following formula: volume $=$ length $\times$ width $\times$ height.

Immunodetection techniques. Conditions for immunoblotting, immunofluorescence, and immunohistochemistry were as previously reported (74). The list of antibodies is provided in Supplemental Table 5. Scans of the full uncut immunoblots are shown in Supplemental Figure 5.

For immunofluorescence, cells were fixed with $4 \%$ paraformaldehyde and permeabilized in $0.1 \%$ Triton X-100, followed by blocking in $5 \%$ donkey serum in PBS. Primary antibodies were incubated at $4^{\circ} \mathrm{C}$ overnight, followed by PBS washes, then incubated with secondary antibodies at RT for 1 hour and mounted in Fluoromount Mounting Medium (MilliporeSigma) after nuclear DAPI staining. Immunofluorescence images were acquired with a Zeiss LSM700 Meta laser-scanning microscope.

For immunofluorescence-guided LCM, frozen tissues were freshly cut and immediately fixed with $75 \%$ ethanol for 30 seconds. Sections were then briefly blocked in $10 \%$ donkey serum in PBS for 2 minutes, followed by incubation with Alexa Fluor 488-conjugated antibodies and propidium iodide (MilliporeSigma) for 2 minutes. Sections were washed by rinsing in PBS, then air-dried before processing using the Arcturus XT Microdissection System (Applied Biosystems).

For immunohistochemistry, cells were fixed with acetone for 3 minutes, followed by blocking of endogenous peroxidase by peroxidase-blocking solution $\left(3 \% \mathrm{H}_{2} \mathrm{O}_{2}\right.$ in PBS) for 10 minutes. Cells were further blocked in $10 \%$ goat serum in PBS for 10 minutes. Primary antibodies were incubated for 1 hour at RT, followed by PBS washes, then incubated with secondary antibodies for 30 minutes at RT. Cells were then incubated for 3 minutes with DAB solution, followed by counterstaining for 4 seconds with Harris Haematoxylin (Leica Biosystems).

For signal quantification, acquired images for each color channel were imported into Image J software and converted into binary images. Intensity was measured using "measurement" or "particle analysis" in areas of interest. All measurements were exported as Microsoft Excel files.

RNA-seq and GSEA. RNA-seq analysis was performed as follows. Total RNA was extracted 5 days after shRNA infection for CSLsilencing cells using Direct-zol RNA Miniprep Kit (Zymo Research). Library preparation using TruSeq Kit (Illumina) was done with $4 \mu \mathrm{g}$ of total RNA. Single read was done on an Illumina HiSeq 2000 sequencer at the Genomic Facility (University of Lausanne). Reads were trimmed by using Trimmomatic (v0.22; http://www.usadellab.org/cms/ ?page=trimmomatic), followed by mapping to the human hg19 reference genome using TopHat (v2.0.8b; https://ccb.jhu.edu/software/ tophat/index.shtml). Gene-expression levels were then evaluated using the HTSeq package (release 0.5.4p1; https://htseq.readthedocs. io/en/release_0.9.1/). The DeSeq2 package (https://bioconductor.org/ packages/release/bioc/html/DESeq2.html) was used to normalize count data, estimate biological variance, and determine differential expression in HKC and SCC cell lines after CSL silencing, generating fold-changes and $P$ values for each class comparison. DAVID software (https://david.ncifcrf.gov/) was used for GO analysis on lists of genes up- and downregulated upon CSL silencing. Lists of genes modulated and their corresponding GO are shown in Supplemental Table 1.

GSEA for RNA-seq expression profiles were performed using GSAA-SeqSP software (gene set association analysis for RNA-seq data with sample permutation) (79) from the Gene Set Association Analysis (GSAA) platform (version GSAA_2.0, http://gsaa.unc.edu/). Curated gene sets were obtained from the Molecular Signatures Database (MSigDB v5.2; http://www.broadinstitute.org/gsea/msigdb/). Gene sets tested in this study are listed in Supplemental Table 2.

ChIP-seq and ChIP-tagmentation. ChIP assays with antibody against CSL versus nonimmune IgG control were performed as previously described (40), followed by assessment of fold enrichment for indicated sites. Primer sequences and antibodies used in this assay are listed in Supplemental Tables 4 and 5, respectively.

For ChIP-seq, DNA was immunoprecipitated from $5 \times 10^{6} \mathrm{HKCs}$ using $10 \mu \mathrm{l} \mathrm{CSL}$ antibody per $10^{6}$ cells, followed by quantification by fluorometry on a Qubit system (Invitrogen). A total of 10 ng DNA was used for library preparation using the NEBNext ChIP-Seq Library Prep Kit (Illumina) according to the manufacturer's recommendations. FASTQ file alignments were done using the Burrows-Wheeler Aligner (http://bio-bwa.sourceforge.net), and peak detection was done using Model-Based Analysis for ChIP-Seq (MACS) software (http://liulab. dfci.harvard.edu/MACS) with default parameters. For a graphic illustration of ChIP-Seq peaks, the Integrative Genomics Viewer (IGV) (http://www.broadinstitute.org/igv) was used with Encyclopedia of DNA Elements (ENCODE) data (http://genome.ucsc.edu/ENCODE) for information on chromatin organization. The list of peaks is shown in Supplemental Table 3.

For ChIP-tagmentation, DNA was immunoprecipitated from $1 \times 10^{6} \mathrm{HKCs}$ using $10 \mu \mathrm{l} \mathrm{CSL}$ antibody per $10^{6}$ cells. Chromatin was tagged with Tn5 transposase (Illumina), followed by decrosslinking, purification, and amplification (14 cycles) using tag-specific primers. Amplified products were diluted 10 times, and $1 \mu \mathrm{l}$ was used for qPCR to determine fold enrichment at indicated sites.

Accession numbers. The RNA-seq and ChIP-seq data sets generated for this study were deposited in the NCBI's Gene Expression Omnibus database (GEO GSE102762 and GSE102761).

Statistics. Data are presented as mean \pm SD or mean \pm SEM among controls and experimental groups, as indicated in the figure legends. Statistical significance between controls and experimental groups was assessed by Student's $t$ test (with Benjamini-Hochberg correction where needed) or 1-way ANOVA (with Dunnett's or Tukey's test), as indicated in the figure legends. ${ }^{*} P<0.05$ was considered statistically significant (unless otherwise indicated).

Study approval. All mouse work was carried out according to Swiss guidelines for the use of laboratory animals, with protocols approved by the veterinary office of Canton de Vaud. All excised human samples were used with the approval of the University of Lausanne and CHUV. Informed consent was obtained as required.

\section{Author contributions}

DAL and SHJ performed experiments and contributed to the analysis of results. PO conducted bioinformatics analysis. CS, MB, and 
$\mathrm{RP}$ provided clinical samples. GPD designed the study and wrote the manuscript with the help of DAL.

\section{Acknowledgments}

We thank P. Khavari for the gift of KDM6B plasmid vectors; M. Bongiovanni, R. Panizzon, and U. Wagner for overseeing clinical samples; C. Missero, K. Lefort, S. Goruppi, and B. Özdemir for critical reading of the manuscript; K. Harshman for RNA-seq and ChIP-seq analysis; X. Xu and M.G. Procopio for help in statistical analysis; and C. Pasche, T. Proust, and A.L. Huguenin for technical help. Work was supported by grants from the Swiss National Science Foundation (310030_156191/1), the NIH (R01AR039190; R01AR064786; content does not necessarily represent the official views of the NIH), the Swiss Cancer League (KFS-3301-08-2013-R), and the European Research Council (26075083).

Address correspondence to: G. Paolo Dotto, Department of Biochemistry, University of Lausanne, Epalinges, CH-1066, Switzerland. Phone: 0041216925720; Email: paolo.dotto@unil.ch.
1. Dotto GP, Rustgi AK. Squamous cell cancers: a unified perspective on biology and genetics. Cancer Cell. 2016;29(5):622-637.

2. Dotto GP. Notch tumor suppressor function. Oncogene. 2008;27(38):5115-5123.

3. Missero C, Di Cunto F, Kiyokawa H, Koff A, Dotto GP. The absence of p21Cip1/WAF1 alters keratinocyte growth and differentiation and promotes ras-tumor progression. Genes Dev. 1996;10(23):3065-3075.

4. Nguyen BC, et al. Cross-regulation between Notch and $\mathrm{p} 63$ in keratinocyte commitment to differentiation. Genes Dev. 2006;20(8):1028-1042.

5. Demehri S, Morimoto M, Holtzman MJ, Kopan R. Skin-derived TSLP triggers progression from epidermal-barrier defects to asthma. PLoS Biol. 2009;7(5):e1000067.

6. Demehri S, Turkoz A, Kopan R. Epidermal Notch1 loss promotes skin tumorigenesis by impacting the stromal microenvironment. Cancer Cell. 2009;16(1):55-66.

7. Dumortier A, et al. Atopic dermatitis-like disease and associated lethal myeloproliferative disorder arise from loss of Notch signaling in the murine skin. PLOS ONE. 2010;5(2):e9258.

8. Casey LM, Lan Y, Cho ES, Maltby KM, Gridley T, Jiang R. Jag2-Notch1 signaling regulates oral epithelial differentiation and palate development. Dev Dyn. 2006;235(7):1830-1844.

9. Ohashi S, et al. NOTCH1 and NOTCH3 coordinate esophageal squamous differentiation through a CSL-dependent transcriptional network. Gastroenterology. 2010;139(6):2113-2123.

10. Rock JR, Gao X, Xue Y, Randell SH, Kong YY, Hogan BL. Notch-dependent differentiation of adult airway basal stem cells. Cell Stem Cell. 2011;8(6):639-648.

11. Talora C, Sgroi DC, Crum CP, Dotto GP. Specific down-modulation of Notch1 signaling in cervical cancer cells is required for sustained HPV-E6/E7 expression and late steps of malignant transformation. Genes Dev. 2002;16(17):2252-2263.

12. Lefort $\mathrm{K}$, et al. Notch 1 is a 53 target gene involved in human keratinocyte tumor suppression through negative regulation of ROCK1/2 and MRCKalpha kinases. Genes Dev. 2007;21(5):562-577.

13. Yugawa T, Handa K, Narisawa-Saito M, Ohno S, Fujita M, Kiyono T. Regulation of Notch1 gene expression by $\mathrm{p} 53$ in epithelial cells. Mol Cell Biol. 2007;27(10):3732-3742.

14. Kopan R, Ilagan MX. The canonical Notch signaling pathway: unfolding the activation mechanism. Cell. 2009;137(2):216-233.
15. Johnson JE, Macdonald RJ. Notch-independent functions of CSL. Curr Top Dev Biol. 2011;97:55-74.

16. Kovall RA, Gebelein B, Sprinzak D, Kopan R. The canonical Notch signaling pathway: structural and biochemical insights into shape, sugar, and force. Dev Cell. 2017;41(3):228-241.

17. Pan $Y$, et al. gamma-secretase functions through Notch signaling to maintain skin appendages but is not required for their patterning or initial morphogenesis. Dev Cell. 2004;7(5):731-743.

18. Campbell RM, Tummino PJ. Cancer epigenetics drug discovery and development: the challenge of hitting the mark. J Clin Invest. 2014;124(1):64-69.

19. Nichol JN, Dupéré-Richer D, Ezponda T, Licht JD, Miller WH. H3K27 Methylation: a focal point of epigenetic deregulation in cancer. Adv Cancer Res. 2016;131:59-95.

20. Ntziachristos $P$, et al. Contrasting roles of histone 3 lysine 27 demethylases in acute lymphoblastic leukaemia. Nature. 2014;514(7523):513-517.

21. Burchfield JS, Li Q, Wang HY, Wang RF. JMJD3 as an epigenetic regulator in development and disease. Int J Biochem Cell Biol. 2015;67:148-157.

22. Perrigue PM, Najbauer J, Barciszewski J. Histone demethylase JMJD3 at the intersection of cellular senescence and cancer. Biochim Biophys Acta. 2016;1865(2):237-244.

23. Salminen A, Kaarniranta K, Hiltunen M, Kauppinen A. Histone demethylase Jumonji D3 (JMJD3/KDM6B) at the nexus of epigenetic regulation of inflammation and the aging process. JMol Med. 2014;92(10):1035-1043.

24. Anderton JA, et al. The H3K27me3 demethylase, KDM6B, is induced by Epstein-Barr virus and over-expressed in Hodgkin's Lymphoma. Oncogene. 2011;30(17):2037-2043.

25. Ramadoss S, Chen X, Wang CY. Histone demethylase KDM6B promotes epithelial-mesenchymal transition. JBiol Chem. 2012;287(53):44508-44517.

26. Perrigue $P M$, et al. The histone demethylase jumonji coordinates cellular senescence including secretion of neural stem cell-attracting cytokines. Mol Cancer Res. 2015;13(4):636-650.

27. Park WY, Hong BJ, Lee J, Choi C, Kim MY. H3K27 Demethylase JMJD3 employs the NF- $\kappa B$ and BMP signaling pathways to modulate the tumor microenvironment and promote melanoma progression and metastasis. Cancer Res. 2016;76(1):161-170.

28. Shen Y, et al. Expression and significance of histone H3K27 demethylases in renal cell carcinoma. BMC Cancer. 2012;12:470.

29. Agger K, et al. The H3K27me3 demethylase JMJD3 contributes to the activation of the
INK4A-ARF locus in response to oncogeneand stress-induced senescence. Genes Dev. 2009;23(10):1171-1176.

30. Pereira F, et al. KDM6B/JMJD3 histone demethylase is induced by vitamin $\mathrm{D}$ and modulates its effects in colon cancer cells. Hum Mol Genet. 2011;20(23):4655-4665.

31. Tokunaga R, et al. The prognostic significance of histone lysine demethylase JMJD3/ KDM6B in colorectal cancer. Ann Surg Oncol. 2016;23(2):678-685.

32. Yamamoto K, et al. Loss of histone demethylase KDM6B enhances aggressiveness of pancreatic cancer through downregulation of C/EBPa. Car cinogenesis. 2014;35(11):2404-2414.

33. Sen GL, Webster DE, Barragan DI, Chang HY, Khavari PA. Control of differentiation in a self-renewing mammalian tissue by the histone demethylase JMJD3. Genes Dev. 2008;22(14):1865-1870.

34. Chen DS, Mellman I. Oncology meets immunology: the cancer-immunity cycle. Immunity. 2013;39(1):1-10.

35. De Santa F, et al. Jmjd 3 contributes to the contro of gene expression in LPS-activated macrophages. EMBO J. 2009;28(21):3341-3352.

36. De Santa F, Totaro MG, Prosperini E, Notarbartolo S, Testa G, Natoli G. The histone H3 lysine-27 demethylase Jmjd3 links inflammation to inhibition of polycomb-mediated gene silencing. Cell. 2007;130(6):1083-1094.

37. Lee K, et al. Molecular mechanism of Jmjd3mediated interleukin- 6 gene regulation in endothelial cells underlying spinal cord injury. JNeurochem. 2012;122(2):272-282.

38. Ishii $\mathrm{M}$, et al. Epigenetic regulation of the alternatively activated macrophage phenotype. Blood. 2009;114(15):3244-3254.

39. Menietti E, Xu X, Ostano P, Joseph JM, Lefort K, Dotto GP. Negative control of CSL gene transcription by stress/DNA damage response and p53. Cell Cycle. 2016;15(13):1767-1778.

40. Procopio MG, et al. Combined CSL and p53 downregulation promotes cancerassociated fibroblast activation. Nat Cell Biol. 2015;17(9):1193-1204.

41. Rangarajan $\mathrm{A}$, et al. Notch signaling is a direct determinant of keratinocyte growth arrest and entry into differentiation. EMBO J. 2001;20(13):3427-3436.

42. Olivier M, Eeles R, Hollstein M, Khan MA, Harris CC, Hainaut P. The IARC TP53 database: new online mutation analysis and recommendations to users. Hum Mutat. 2002;19(6):607-614. 
43. Aster JC, Robertson ES, Hasserjian RP, Turner JR, Kieff E, Sklar J. Oncogenic forms of NOTCH1 lacking either the primary binding site for RBP-Jkappa or nuclear localization sequences retain the ability to associate with RBPJkappa and activate transcription. J Biol Chem. 1997;272(17):11336-11343.

44. Wu L, Aster JC, Blacklow SC, Lake R, ArtavanisTsakonas S, Griffin JD. MAML1, a human homologue of Drosophila mastermind, is a transcriptional co-activator for NOTCH receptors. Nat Genet. 2000;26(4):484-489.

45. Barrandon Y, Green H. Three clonal types of keratinocyte with different capacities for multiplication. Proc Natl Acad Sci U S A. 1987;84(8):2302-2306.

46. Pastrana E, Silva-Vargas V, Doetsch F. Eyes wide open: a critical review of spher10-formation as an assay for stem cells. Cell Stem Cell. 2011;8(5):486-498.

47. Harper LJ, Piper K, Common J, Fortune F, Mackenzie IC. Stem cell patterns in cell lines derived from head and neck squamous cell carcinoma. J Oral Pathol Med. 2007;36(10):594-603.

48. Rocco JW, Leong CO, Kuperwasser N, DeYoung MP, Ellisen LW. p63 mediates survival in squamous cell carcinoma by suppression of p73-dependent apoptosis. Cancer Cell. 2006;9(1):45-56.

49. Subramanian A, et al. Gene set enrichment analysis: a knowledge-based approach for interpreting genome-wide expression profiles. Proc Natl Acad Sci U S A. 2005;102(43):15545-15550.

50. Zhao L, Xia J, Wang X, Xu F. Transcriptional regulation of CCL2O expression. Microbes Infect. 2014;16(10):864-870.

51. Kolev V, et al. EGFR signalling as a negative regulator of Notch1 gene transcription and function in proliferating keratinocytes and cancer. Nat Cell Biol. 2008;10(8):902-911.

52. Moriyama M, et al. Multiple roles of Notch signaling in the regulation of epidermal development. Dev Cell. 2008;14(4):594-604.

53. Blanpain C, Lowry WE, Pasolli HA, Fuchs E. Canonical notch signaling functions as a commitment switch in the epidermal lineage. Genes Dev. 2006;20(21):3022-3035.

54. Kulic I, et al. Loss of the Notch effector RBPJ promotes tumorigenesis. JExp Med. 2015;212(1):37-52.

55. Lv Q, Shen R, Wang J. RBPJ inhibition impairs the growth of lung cancer. Tumour Biol.
2015;36(5):3751-3756.

56. Xue L, et al. Inhibition of recombining binding protein suppressor of hairless (RBPJ) impairs the growth of prostate cancer. Cell Physiol Biochem. 2015;36(5):1982-1990.

57. Yong T, Sun A, Henry MD, Meyers S, Davis JN. Down regulation of CSL activity inhibits cell proliferation in prostate and breast cancer cells. JCell Biochem. 2011;112(9):2340-2351.

58. Demehri S, et al. Notch-deficient skin induces a lethal systemic B-lymphoproliferative disorder by secreting TSLP, a sentinel for epidermal integrity. PLoS Biol. 2008;6(5):e123.

59. Hunter CA, Jones SA. IL- 6 as a keystone cytokine in health and disease. Nat Immunol. 2015;16(5):448-457.

60. Kuilman T, et al. Oncogene-induced senescence relayed by an interleukin-dependent inflammatory network. Cell. 2008;133(6):1019-1031.

61. Li Q, et al. Stage-dependent and locus-specific role of histone demethylase Jumonji D3 (JMJD3) in the embryonic stages of lung development. PLoS Genet. 2014;10(7):e1004524.

62. Satoh T, et al. The Jmjd3-Irf4 axis regulates M2 macrophage polarization and host responses against helminth infection. Nat Immunol. 2010;11(10):936-944.

63. Ma J, et al. KDM6B elicits cell apoptosis by promoting nuclear translocation of FOXO1 in non-small cell lung cancer. Cell Physiol Biochem. 2015;37(1):201-213.

64. Cloos PA, Christensen J, Agger K, Helin K. Erasing the methyl mark: histone demethylases at the center of cellular differentiation and disease. Genes Dev. 2008;22(9):1115-1140.

65. González-Ramírez I, Soto-Reyes E, SánchezPérez Y, Herrera LA, García-Cuellar C. Histones and long non-coding RNAs: the new insights of epigenetic deregulation involved in oral cancer. Oral Oncol. 2014;50(8):691-695.

66. Nigro JM, et al. Mutations in the p53 gene occur in diverse human tumour types. Nature. 1989;342(6250):705-708.

67. Solá S, et al. p53 interaction with JMJD3 results in its nuclear distribution during mouse neural stem cell differentiation. PLOS ONE. 2011;6(3):e18421.

68. Ene CI, et al. Histone demethylase Jumonji D3 (JMJD3) as a tumor suppressor by regulating p53 protein nuclear stabilization. PLOS ONE. 2012;7(12):e51407.

69. Pham D, Yu Q, Walline CC, Muthukrishnan R,
Blum JS, Kaplan MH. Opposing roles of STAT4 and Dnmt3a in Th1 gene regulation. J Immunol. 2013;191(2):902-911.

70. Przanowski P, et al. The signal transducers Stat1 and Stat 3 and their novel target Jmjd 3 drive the expression of inflammatory genes in microglia. JMol Med. 2014;92(3):239-254.

71. Estarás C, Akizu N, García A, Beltrán S, de la Cruz $\mathrm{X}$, Martínez-Balbás MA. Genome-wide analysis reveals that Smad3 and JMJD3 HDM co-activate the neural developmental program. Development. 2012;139(15):2681-2691.

72. Shan J, Fu L, Balasubramanian MN, Anthony T, Kilberg MS. ATF4-dependent regulation of the JMJD3 gene during amino acid deprivation can be rescued in Atf4-deficient cells by inhibition of deacetylation. J Biol Chem. 2012;287(43):36393-36403.

73. Lee HY, Choi K, Oh H, Park YK, Park H. HIF-1dependent induction of Jumonji domaincontaining protein (JMJD) 3 under hypoxic conditions. Mol Cells. 2014;37(1):43-50.

74. Hu B, et al. Multifocal epithelial tumors and field cancerization from loss of mesenchymal CSL signaling. Cell. 2012;149(6):1207-1220.

75. Kruidenier L, et al. A selective jumonji H3K27 demethylase inhibitor modulates the proinflammatory macrophage response. Nature. 2012;488(7411):404-408.

76. Wu G, et al. The genomic landscape of diffuse intrinsic pontine glioma and pediatric non-brainstem high-grade glioma. Nat Genet. 2014;46(5):444-450.

77. Hashizume R, et al. Pharmacologic inhibition of histone demethylation as a therapy for pediatric brainstem glioma. Nat Med. 2014;20(12):1394-1396.

78. Brooks YS, et al. Multifactorial ER $\beta$ and NOTCH1 control of squamous differentiation and cancer. J Clin Invest. 2014;124(5):2260-2276.

79. Xiong Q, Mukherjee S, Furey TS. GSAASeqSP: a toolset for gene set association analysis of RNASeq data. Sci Rep. 2014;4:6347.

80. Cao C, et al. SIRT1 confers protection against UVB- and $\mathrm{H} 2 \mathrm{O} 2$-induced cell death via modulation of p53 and JNK in cultured skin keratinocytes. J Cell Mol Med. 2009;13(9B):3632-3643.

81. Barrow JR. Wnt/PCP signaling: a veritable polar star in establishing patterns of polarity in embryonic tissues. Semin Cell Dev Biol. 2006;17(2):185-193. 